\title{
The technique of robotic anatomic pulmonary segmentectomy II: left sided segments
}

\author{
Farid Gharagozloo, Mark Meyer \\ Center for Advanced Thoracic Surgery, Global Robotics Institute, Advent Health Celebration, University of Central Florida, \\ Celebration, FL 34786, USA.
}

Correspondence to: Dr. Farid Gharagozloo, Center for Advanced Thoracic Surgery, Global Robotics Institute, Advent Health Celebration, University of Central Florida, 400 Celebration Place, Celebration, FL 34786, USA.

E-mail: farid.gharagozloo.md@adventhealth.com

How to cite this article: Gharagozloo F, Meyer M. The technique of robotic anatomic pulmonary segmentectomy II: left sided segments. Mini-invasive Surg 2020;4:68. http://dx.doi.org/10.20517/2574-1225.2020.60

Received: 17 Jun 2020 First Decision: 24 Jul 2020 Revised: 26 Jul 2020 Accepted: 30 Jul 2020 Published: 12 Oct 2020

Academic Editor: Noriyoshi Sawabata Copy Editor: Cai-Hong Wang Production Editor: Jing Yu

\begin{abstract}
Anatomic pulmonary segmentectomy and mediastinal nodal dissection has been advocated in patients with smaller tumors or patients with limited pulmonary reserve. The overall 5-year survival and the lung cancer-specific 5-year survival following anatomic segmentectomy have been shown to be equivalent to that of lobectomy. Robotic surgical systems have the advantage of magnified, high-definition three-dimensional visualization and greater instrument maneuverability in a minimally invasive platform. These robotic systems can facilitate the dissection of the bronchovascular structures and replicate the technique of segmentectomy by thoracotomy. Greater experience with the robotic platform has resulted in a reproducible anatomic segmentectomy technique. This is a companion paper to The Technique of Robotic Anatomic Segmentectomy I: Right Sided Segments. This paper outlines the technique of anatomic pulmonary segmentectomy for the left lung: Left Upper Lobe (LUL) Anterior Segment (S3), LUL Apicoposterior Segment (S1 + S2), LUL Lingulectomy (S4, S5), Left Lower Lobe (LLL) Superior Segmentectomy (S6), and LLL Basal Segmentectomy (S7-S10).
\end{abstract}

Keywords: Robotic, segmentectomy, lung cancer, superior segment, anterior segment, apicoposterior segment, basal segment, sublobar resection

\section{INTRODUCTION}

Historically, anatomic pulmonary segmentectomy was used for the surgical treatment of lung abscesses and other lung infections. Chevalier Jackson and John Hubert first proposed a system of nomenclature for the

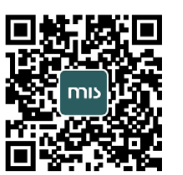


bronchopulmonary segments ${ }^{[1]}$. In 1939, Churchill and Belsey ${ }^{[2]}$ reported the first anatomic segmentectomy, a lingulectomy. Edward Boyden described the vascular and bronchial anatomy for pulmonary segments ${ }^{[3]}$.

In the latter half of the twentieth century, the advent of antibiotic therapy led to a decrease in segmentectomies performed for infectious lung processes and an increase in their use for primary malignancies of the lung. In the 1960's and 1970's, Rasmussen and Clagett published reports of segmentectomy for lung cancer with low mortality ${ }^{[4]}$. With the introduction of stapling devices in the late 1960's, wedge resections, which were technically much easier, became widely used. Thereafter and unfortunately, wedge resection, a nonanatomic pulmonary resection, and individual ligation anatomic segmentectomy became grouped as "sublobar resections". Subsequent studies showed that anatomic segmentectomy was associated with significantly better cancer-related survival than wedge resection ${ }^{[5]}$. However, as anatomic segmentectomy is a technically more demanding procedure than lobectomy, lobectomy became the procedure of choice for early stage lung cancer.

Recently, anatomic pulmonary segmentectomy has been shown to be a viable oncologic procedure for early lung cancer, including patients who are elderly or have limited pulmonary reserve ${ }^{[6-14]}$. As a result of high definition three-dimensional visualization and increased maneuverability of the surgical instruments in a small space, the surgical robot has the distinct advantage of replicating the technique of anatomic segmentectomy by thoracotomy using a minimally invasive platform ${ }^{[15]}$. Although there has been skepticism about the cost and the lack of evidence of the survival advantage of using robotic lobectomy, the robotic platform seems to be especially suited to a minimally invasive approach to anatomic segmentectomy ${ }^{[15,16]}$. Greater experience with the robotic platform has resulted in a reproducible anatomic segmentectomy technique.

This is a companion paper to The Technique of Robotic Anatomic Segmentectomy I: Right Sided Segments. This paper outlines the technique of anatomic pulmonary segmentectomy for the left lung: Left Upper Lobe (LUL) Anterior Segment (S3), LUL Apicoposterior Segment (S1 + S2), LUL Lingulectomy (S4, S5), Left Lower Lobe (LLL) Superior Segmentectomy (S6), and LLL Basal Segmentectomy (S7-S10).

\section{ANATOMIC SEGMENTECTOMY IN THE LEFT LUNG}

The bronchopulmonary segments of the left lower lobe are similar to the right lower lobe. Although there are only two lobes in the left lung, there is some symmetry among the bronchopulmonary segments bilaterally. However, some segments of the left lung merge, resulting in fewer bronchopulmonary segments on the left than there are on the right lung [Figure 1].

The apicoposterior segment $\left(S_{1}+S_{2}\right)$ of the left upper lobe represents the fusion of the apical and posterior segments. Although the Lingula is divided into two bronchopulmonary segments, the superior (S4) and inferior (S5) Lingular segments, from a practical standpoint, $\mathrm{S} 4+\mathrm{S} 5$ segmentectomy or lingulectomy is typically performed. In the left lower lobe, there are four segments unlike the right lower lobe which has five segments. The anteromedial basal segment $(S 7+S 8)$ represents the fusion of the anterior basal and medial basal segments. The other segments (superior S6, posterior basal S10, and lateral basal S9) maintain the same relative positions as observed in the right lung.

From a surgical standpoint, sublobar resection is usually performed for LUL anterior segment (S3), LUL apicoposterior segment $\left(S_{1}+\right.$ S2), LUL lingulectomy (S4, S5), LLL superior segmentectomy (S6), and LLL basal segmentectomy (S7-S10). It is possible to perform individual anatomic segmentectomy of the basal segments $\mathrm{S} 7+$ S8, S9, or S10. We have no experience with robotic segmentectomy of these individual basal segments and therefore have not included them in this report. 

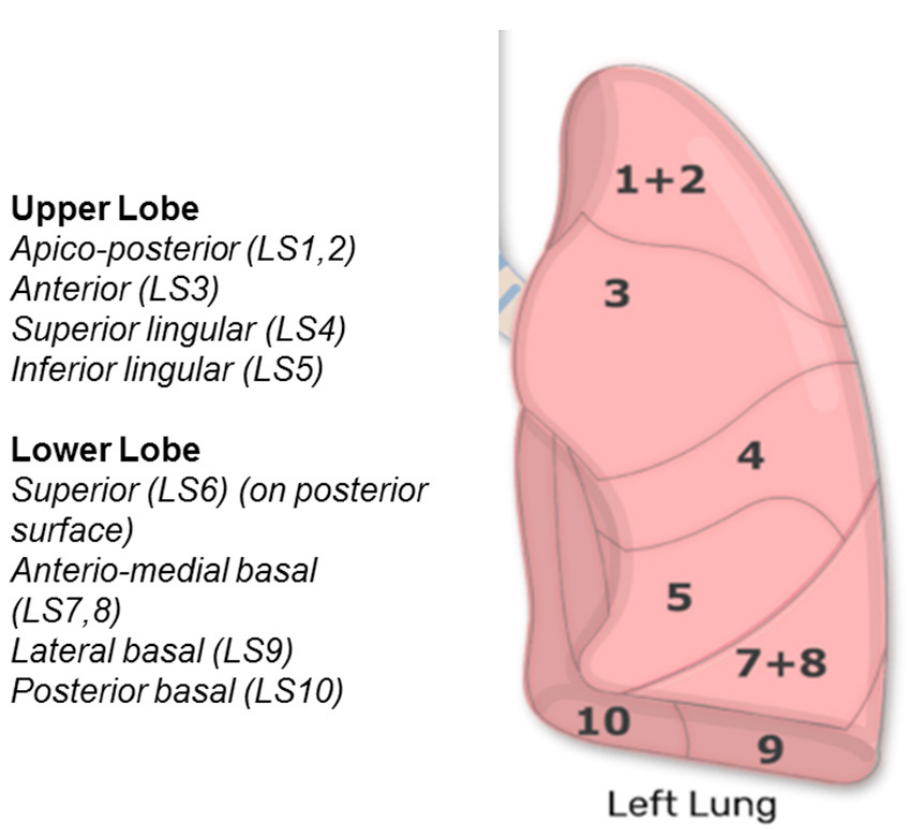

Figure 1. Bronchopulmonary segments of the left lung

\section{Port placement}

The operating room table is reversed such that the pedestal does not interfere with the docking of the robot over the head of the patient.

A double lumen endotracheal tube is placed, and the patient is positioned in a full lateral decubitus position. The left arm is placed over pillows and positioned high enough such that access to the 4th intercostal space in the anterior axillary line is readily attained. The table is flexed in order to move the hip down and to open the intercostal spaces. The lung is deflated and placed on suction. The position of the double lumen tube is rechecked after the patient is prepped and draped. We prefer the use of a double lumen tube as opposed to a bronchial blocker. During robotic dissection, manipulation of the hilum and the bronchus can result in dislodgement of the blocker and loss of lung isolation. Every effort should be made to ensure lung isolation for the entire procedure. The position of the robot over the head of the patient makes manipulation of the endotracheal tube difficult. Untimely inflation of the lung can result in loss of exposure and its associated complications.

Proper port positioning is crucial and a fundamental prerequisite to the conduct of the procedure. Figures 2 and 3 show port placements. A line is drawn from the tip of the scapula to the costal arch. This delineates the highest point in the chest and the midscapular line (posterior axillary line). Pleural entry is with a Hassan needle. Saline is infused and care is taken to look for easy egress of the saline from the needle. If there is concern of pleural adhesions, we use a Visiport Instrument (Medtronic Inc. Norwalk, CT) for entry into the pleural space under direct vision. If the Visiport is used, a purse string is placed in the muscle layer and tied around the robot camera port in order to prevent $\mathrm{CO}_{2}$ leakage. Port \#1 is the camera port. Warm, humidified $\mathrm{CO}_{2}$ is insufflated through this port at a flow rate of $6 \mathrm{~L} / \mathrm{min}$ to a pressure of $6-8 \mathrm{mmHg}$ in order to push the lung and diaphragm away. The other ports are placed under direct vision. Port \#2 is placed in the 7th intercostal space in the posterior scapular line. This port is $9 \mathrm{~cm}$ posterior to Port \#1. Prior to the placement of Port \#3, a 21-gauge needle is inserted into the 7th intercostal space at costovertebral junction from the patient's back and a $10 \mathrm{~mL}$ subpleural bubble of $0.25 \%$ bupivacaine with epinephrine is injected near the intercostal nerve. Next, Port \#3 is placed $9 \mathrm{~cm}$ posterior to Port \#2 in the 7th intercostal space just medial to the spine. Port \#4 is placed $9 \mathrm{~cm}$ anterior to Port \#1 in the 7 th intercostal space at the anterior 


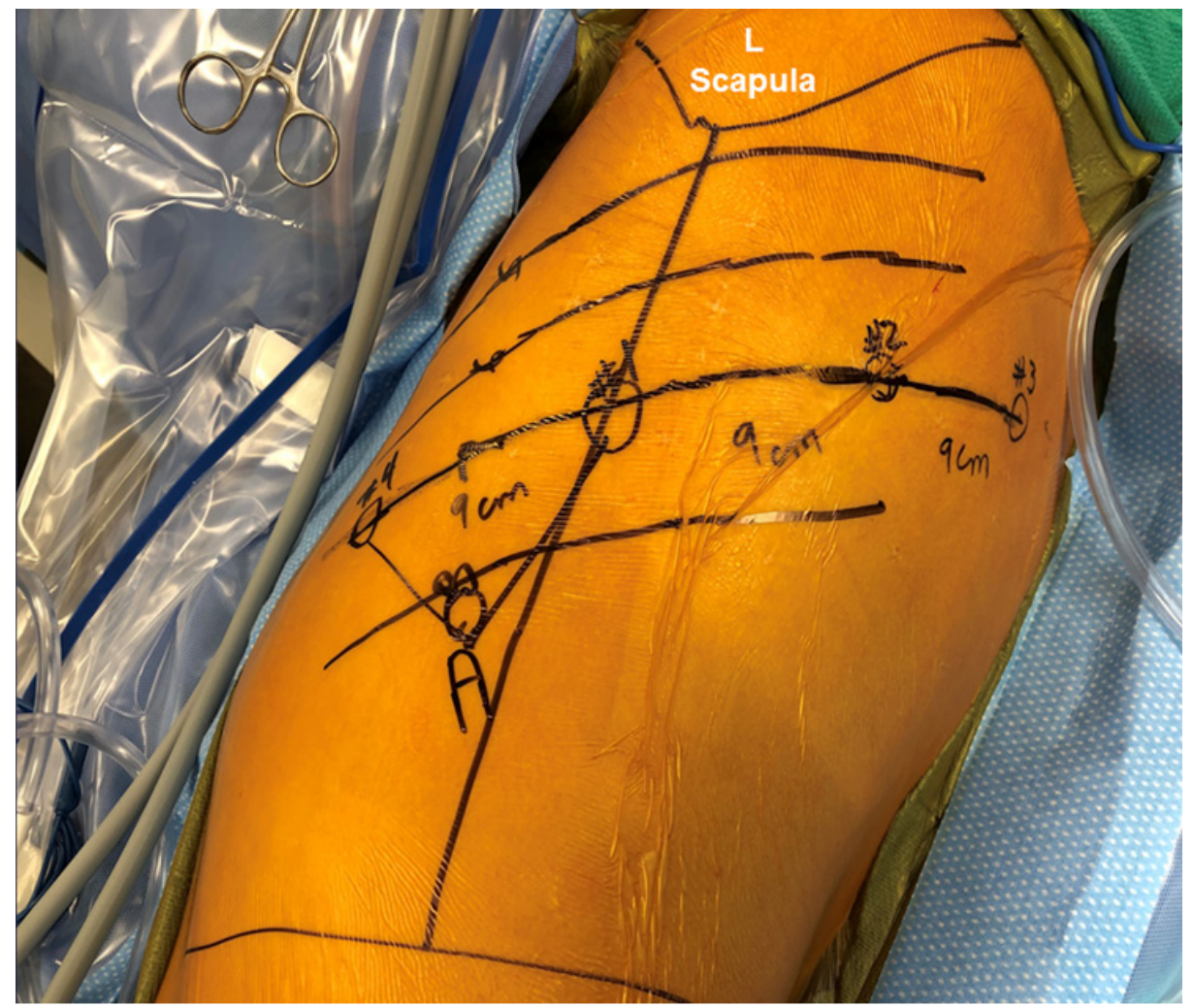

Figure 2. Port placement for robotic segmentectomy in the left chest (please see description in the text)

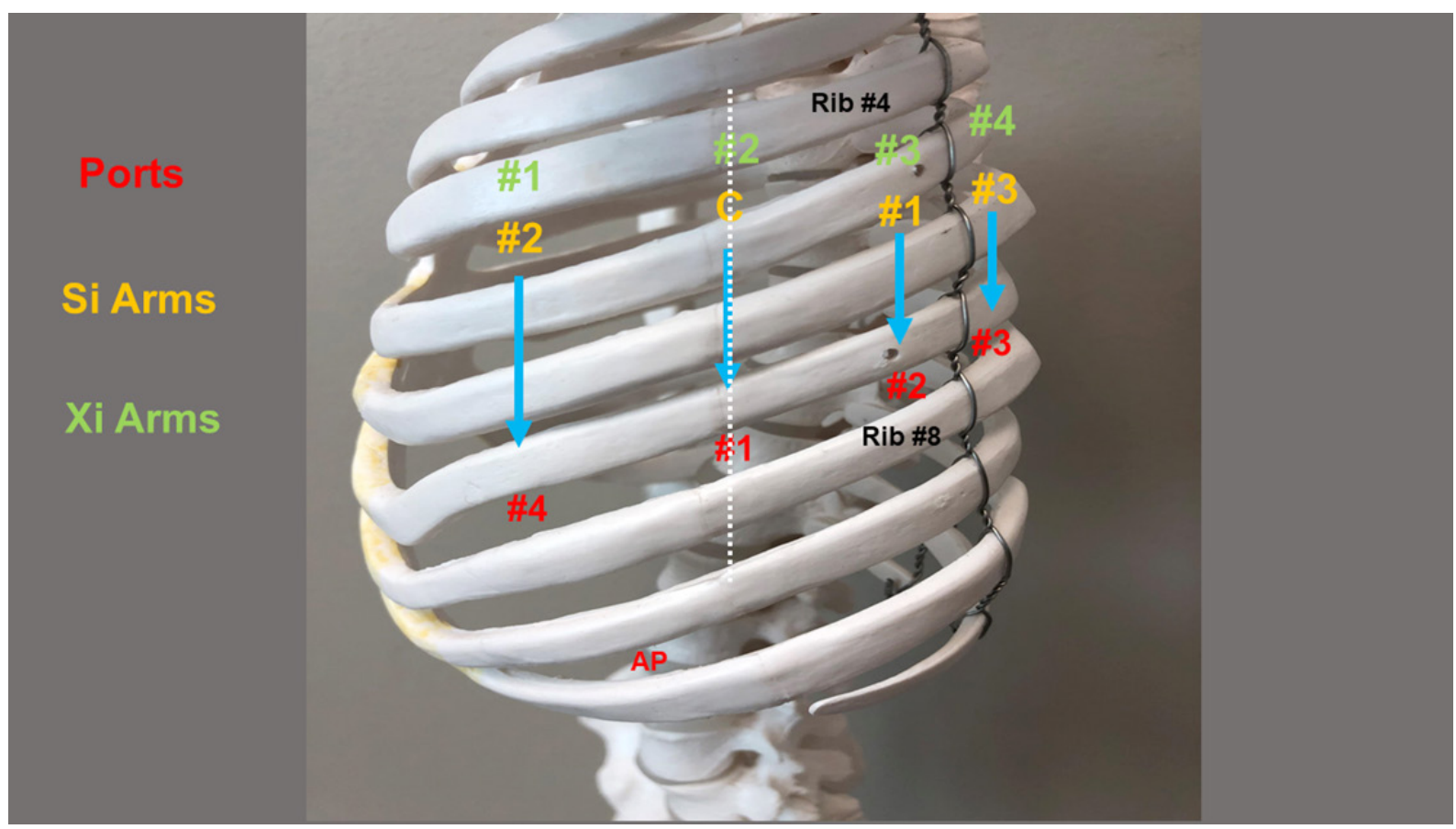

Figure 3. Port placement for robotic segmentectomy in the left chest. Dotted line: Scapular line; Red: Ports; Yellow: Si arm numbering; Green: Xi arm numbering; AP: assistant port 


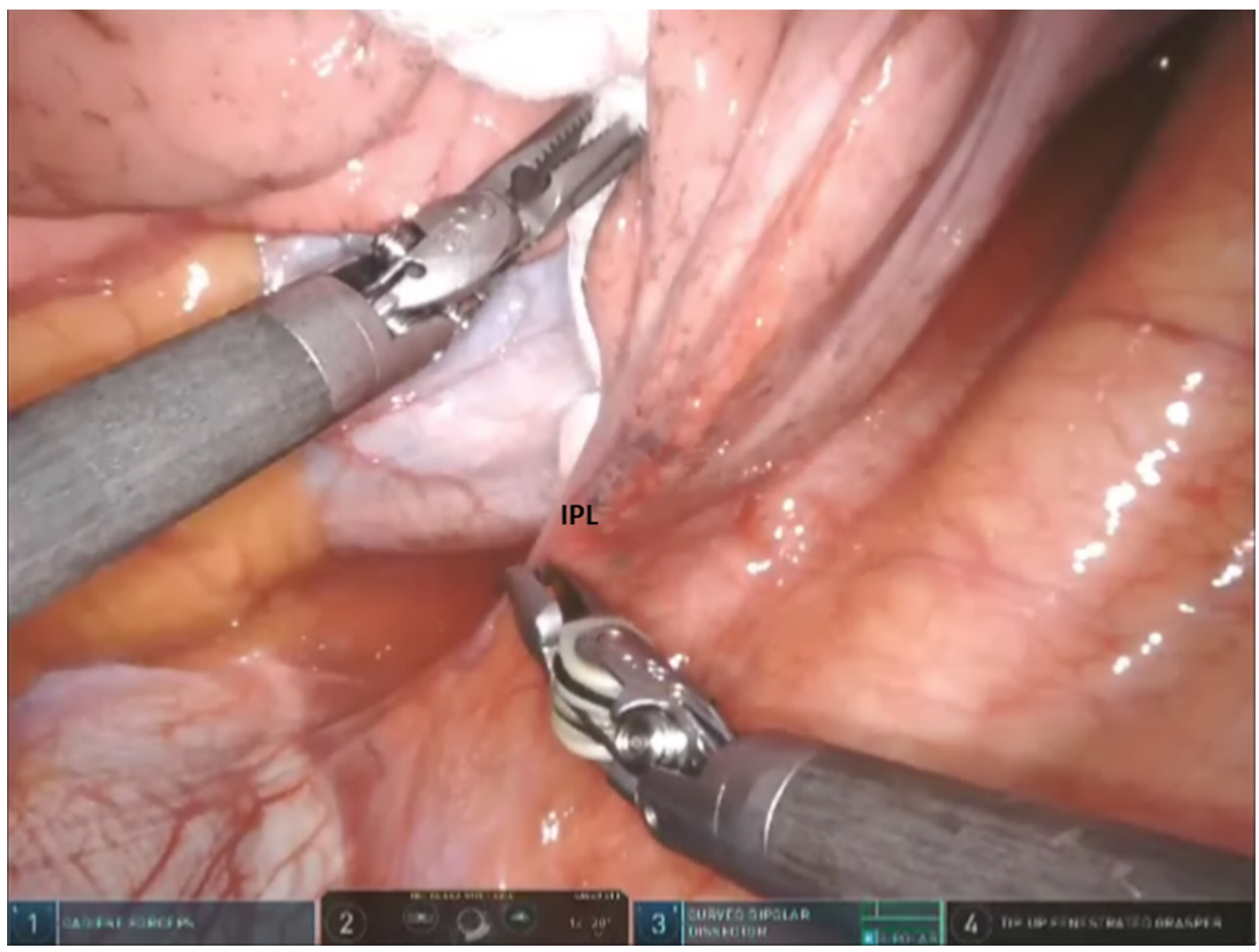

Figure 4. Left sided Segmentectomy: begin by dividing the IPL. IPL: inferior pulmonary ligament

scapular line. The Assistant Port \#5 uses a 10-12 Versiport trocar and is placed in the 9th intercostal space and is triangulated between Port \#1 and \#4.

For the da Vinci Si robot, the bed is angled posteriorly away from the anesthesia machine and the robot is brought in over the head of the patient. For the Xi system, the robot is brought in from the back and perpendicular to the patient and the boom is rotated to the proper position.

One of the advantages of the $\mathrm{Xi}$ robot is that the surgeon can control the stapling device. We prefer a 30 $\mathrm{mm}$ stapler with a white load for the vascular structures, and a blue or green load for the bronchus and the lung tissue as judged by the size and thickness of the structure.

Instruments: $0^{\circ}$ and/or $30^{\circ}$ down viewing endoscope, $5 \mathrm{~mm}$ Thoracic Grasper, Cadiere Forceps, and Curved Bipolar Dissector.

For all segmentectomies, begin by dividing the inferior pulmonary ligament and remove station \#9, and station \#8 [Figures 4 and 5]. The lung is retracted medially and anteriorly in order to remove lymph nodes from station \#7. We find that pulling the nasogastric tube back above the area of subcarinal dissections opens the mediastinal space and facilitates the subcarinal and mediastinal dissection. After the mediastinal dissection, the nasogastric tube is advanced back into the stomach, placed on suction, and used to decompress the stomach and prevent gastric distension and the resultant elevation of the left hemidiaphragm. Next, open the pleura anterior to the vagus nerve. Identify the left mainstem bronchus 


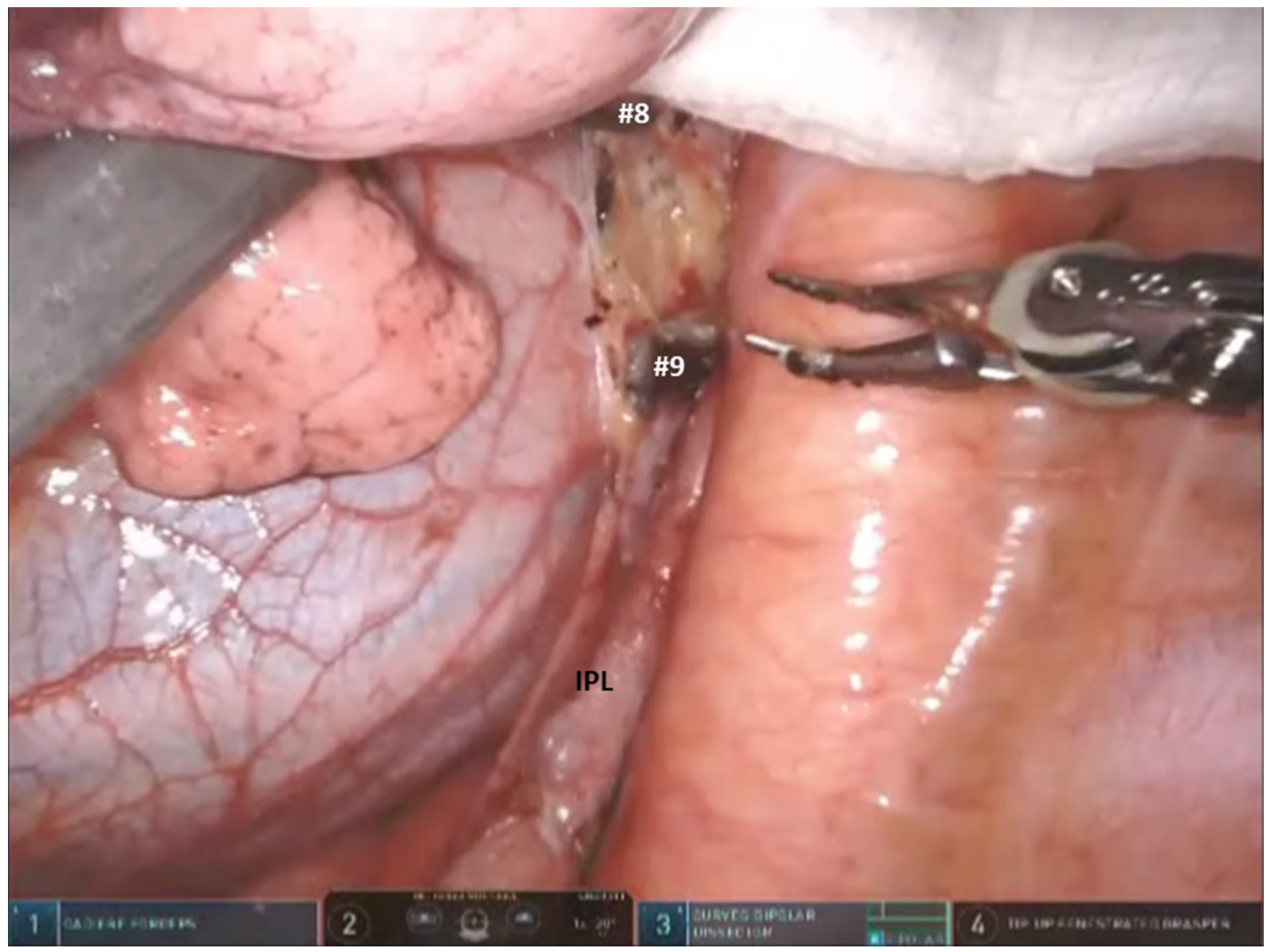

Figure 5. Left sided Segmentectomy: dissection and removal of station \#9 and station \#8 lymph nodes. IPL: inferior pulmonary ligament

and stay inferior to the edge of the cartilage. The station \#7 nodal bundle is accessed between the inferior pulmonary vein and the left mainstem bronchus. The nodal bundle is traced to the carina and is then removed [Figure 6]. Next, the lung is retracted inferiorly, and pleura overlying the station \#5 nodal bundle is opened. Station \#5 nodes are removed [Figure 7].

The left main pulmonary artery is identified above the left main bronchus. The space between the pulmonary artery and the bronchus is opened and station \#10L nodal bundle is identified overlying the superior border of the bronchus [Figure 8]. The space between the pulmonary artery and the aorta is cleared in order to visualize the nodal bundle which encases the apicoposterior trunk of the artery [Figure 9]. Care is taken to identify and preserve the vagus nerve and the recurrent laryngeal branch. After exposing the apicoposterior trunk, the station \#10 nodal bundle is swept in an inferomedial direction, thereby exposing the underside of the truncus branch and its takeoff from the main pulmonary artery.

Next the upper and lower lobe are retracted in opposite directions and the fissure is identified. Dissection of nodal bundle in station \#11 allows for the identification of the pulmonary artery in the fissure [Figure 10]. The artery is most superficial at the junction of the Lingula, upper lobe and the lower lobe. The subadventitial plane is entered, and dissection is carried posteriorly, under the pulmonary parenchyma in the posterior aspect of the fissure toward the main pulmonary artery. The Cadiere forceps is used to pass a vessel loop under the pulmonary parenchyma in the posterior aspect of the fissure. A stapler with a blue cartridge is used to divide the tissue in the posterior aspect of the fissure [Figure 11]. 


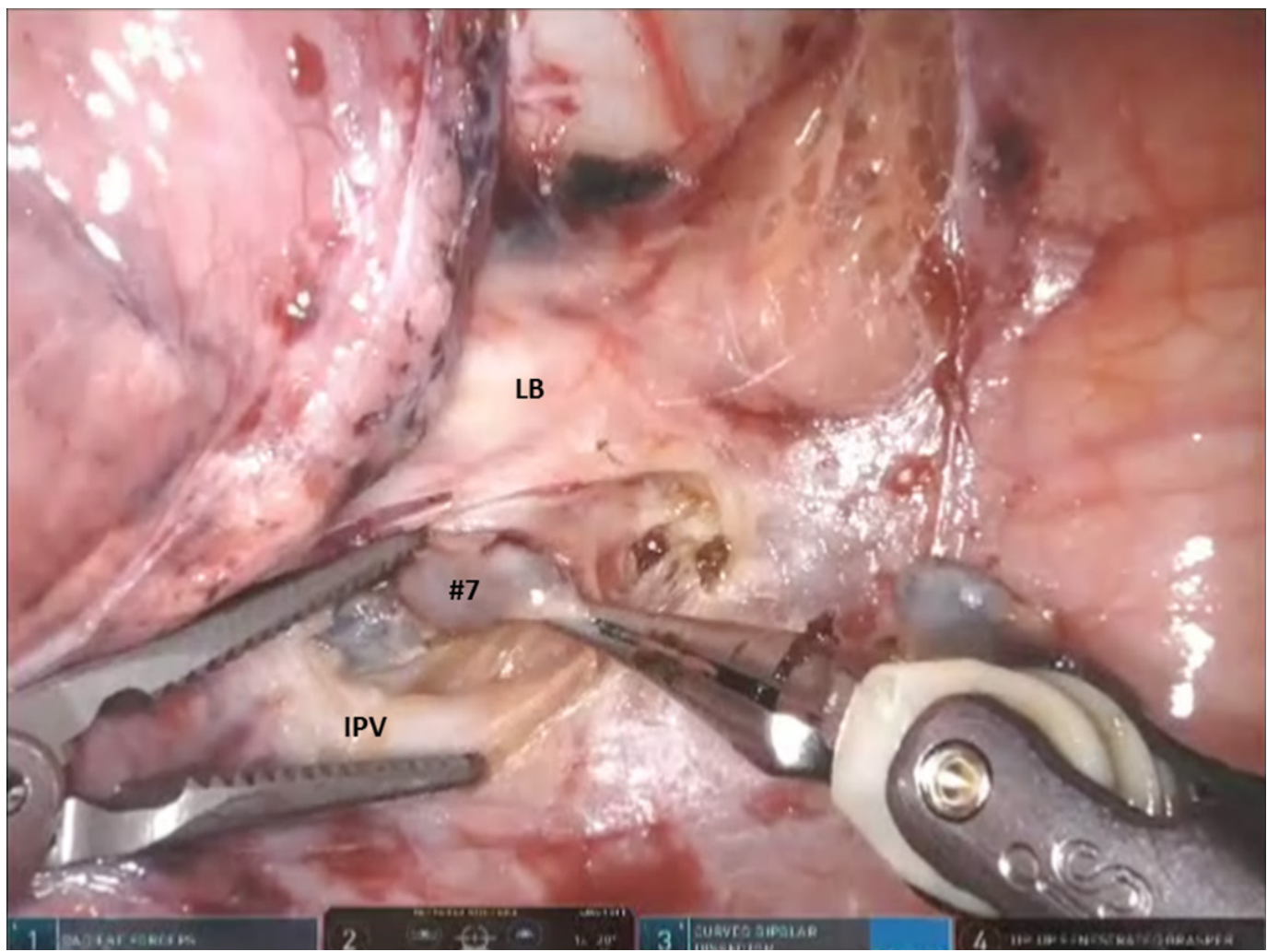

Figure 6. Left sided Segmentectomy: the station \#7 nodal bundle is accessed between the IPV and the LB. IPV: inferior pulmonary vein; LB: left mainstem bronchus

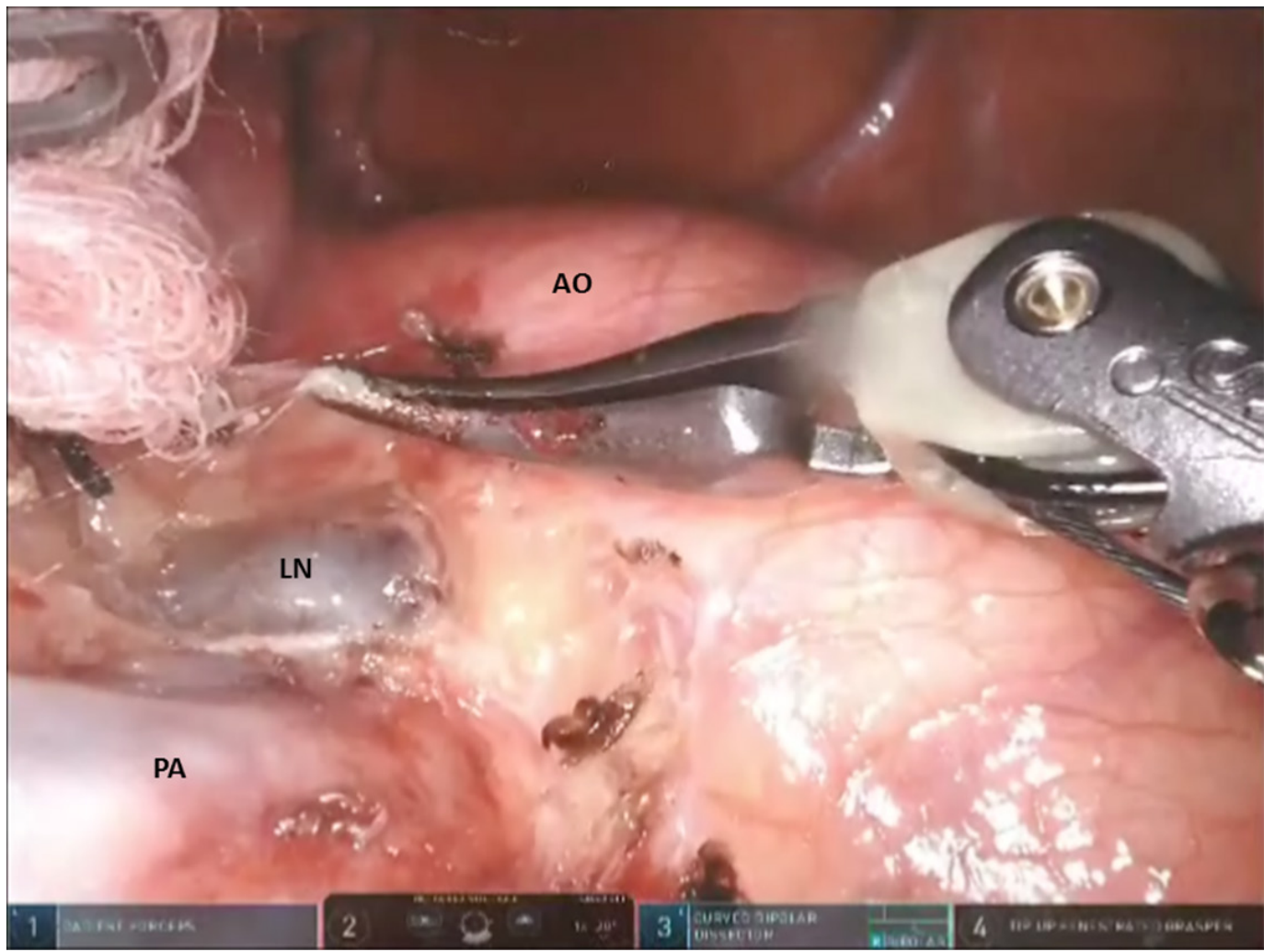

Figure 7. Left sided Segmentectomy: station \#5L nodes are removed from the aorto-pulmonary window. AO: aorta; PA: pulmonary artery; LN: lymph node 


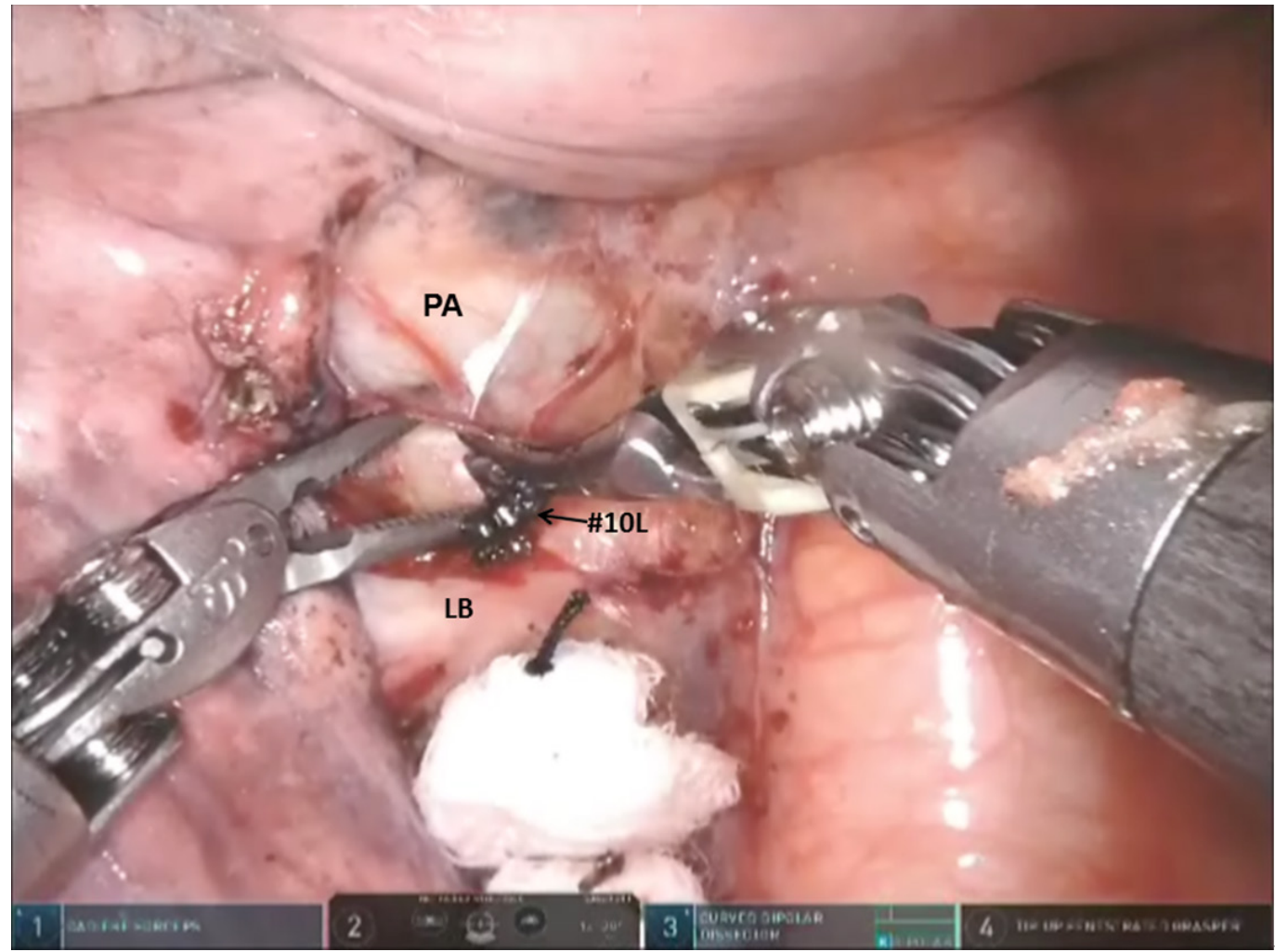

Figure 8. Left sided Segmentectomy: the space between the PA and the LB is opened and the station \#10L nodal bundle is identified overlying the superior border of the bronchus. PA: pulmonary artery; LB: left mainstem bronchus

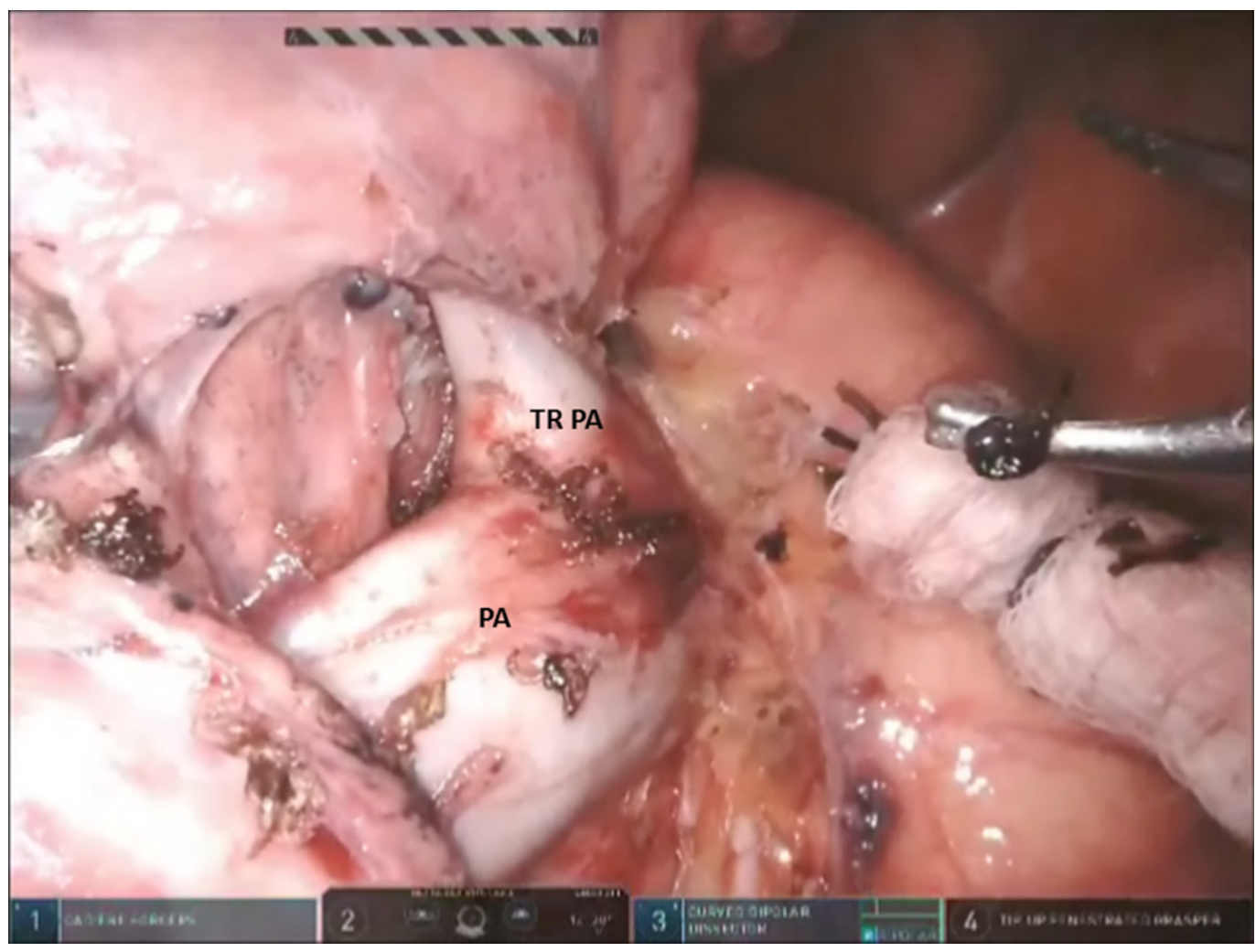

Figure 9. Left sided Segmentectomy: clear the nodal bundle which encases the apicoposterior trunk (TRPA) of the left PA. TRPA: truncus branch of pulmonary artery; PA: pulmonary artery 


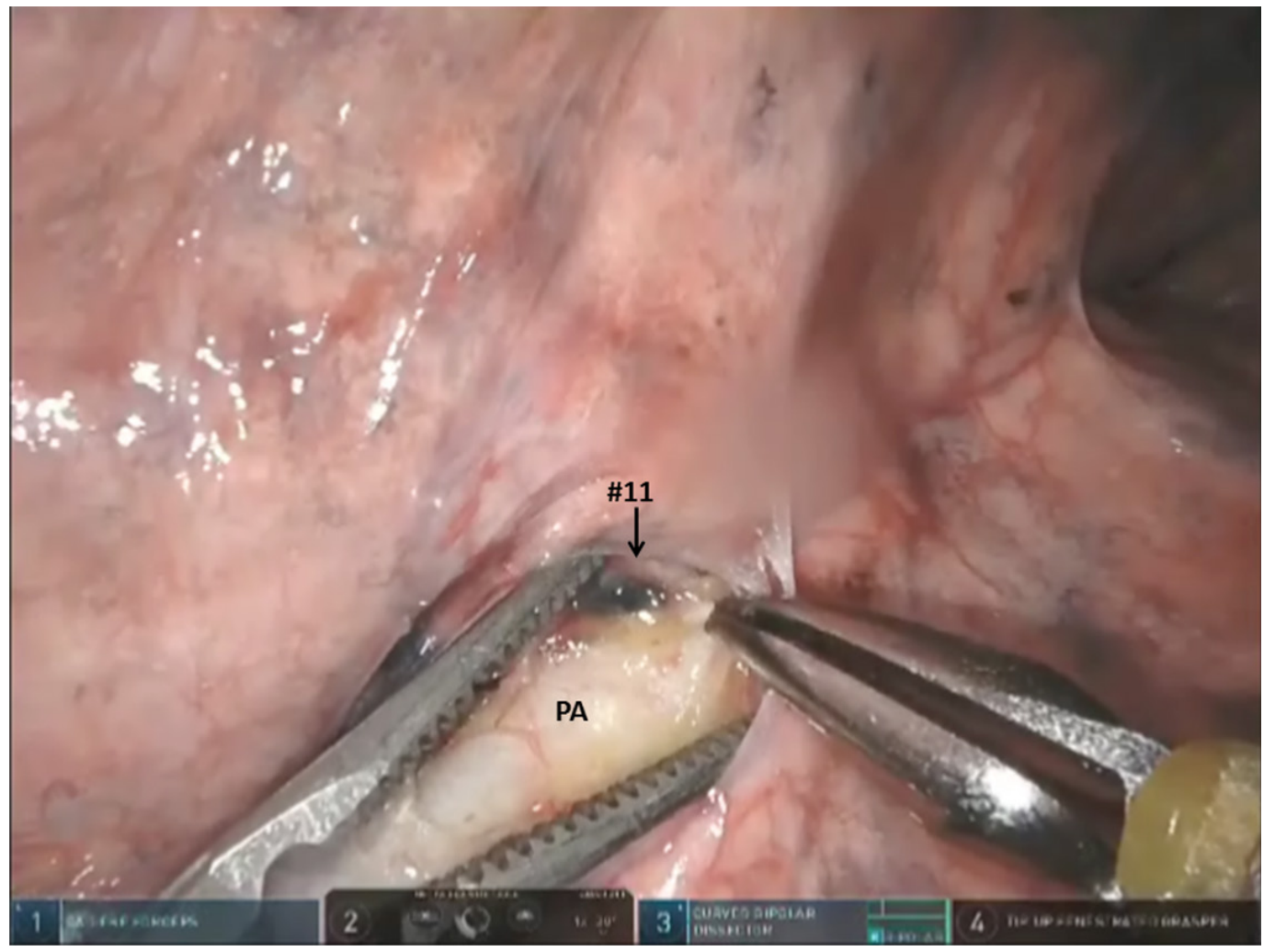

Figure 10. Left sided Segmentectomy: dissection of nodal bundle in station \#11 allows for the identification of the PA in the fissure. PA: pulmonary artery

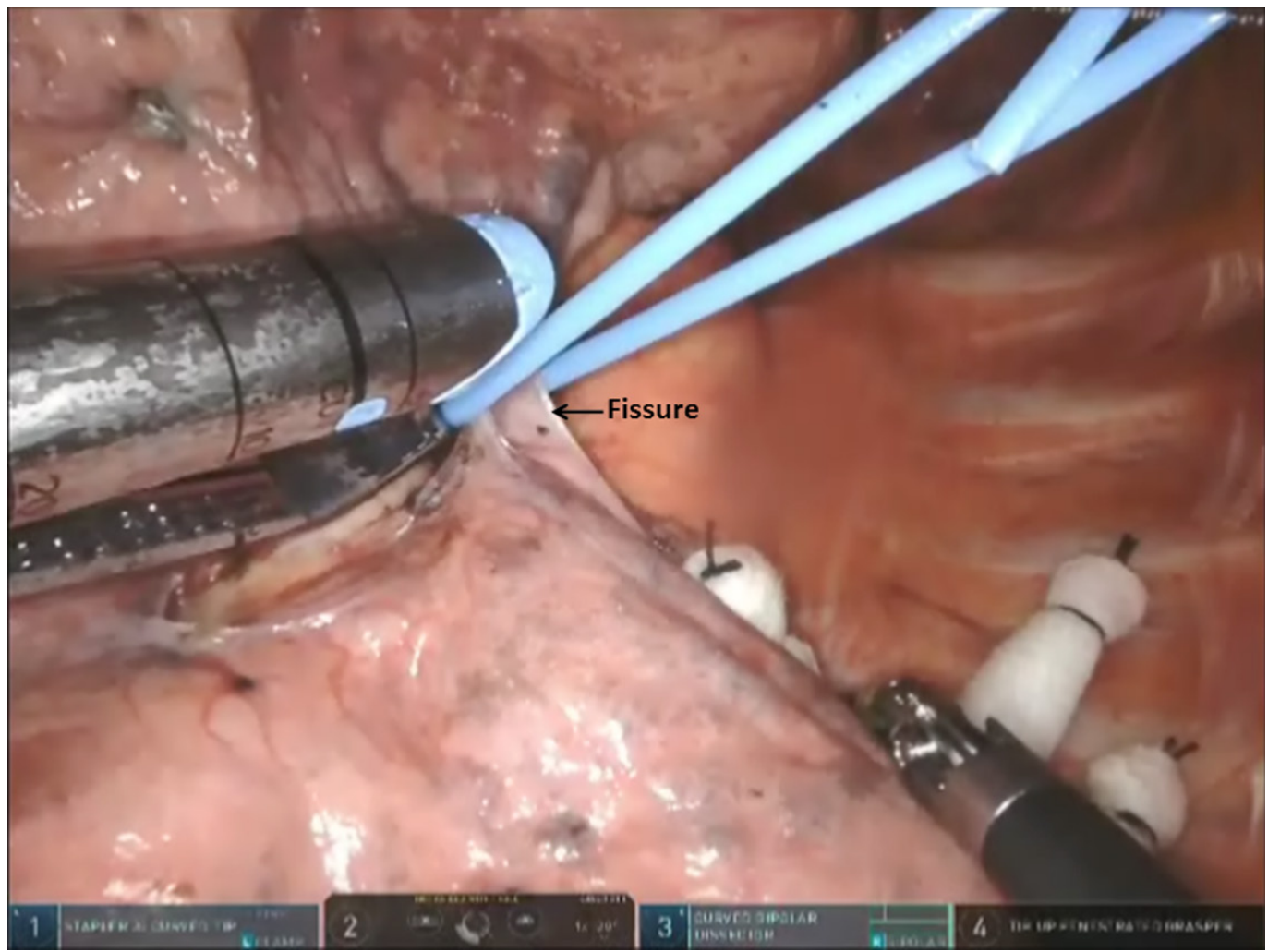

Figure 11. Left sided Segmentectomy: pass a vessel loop under the pulmonary parenchyma in the posterior aspect of the fissure 


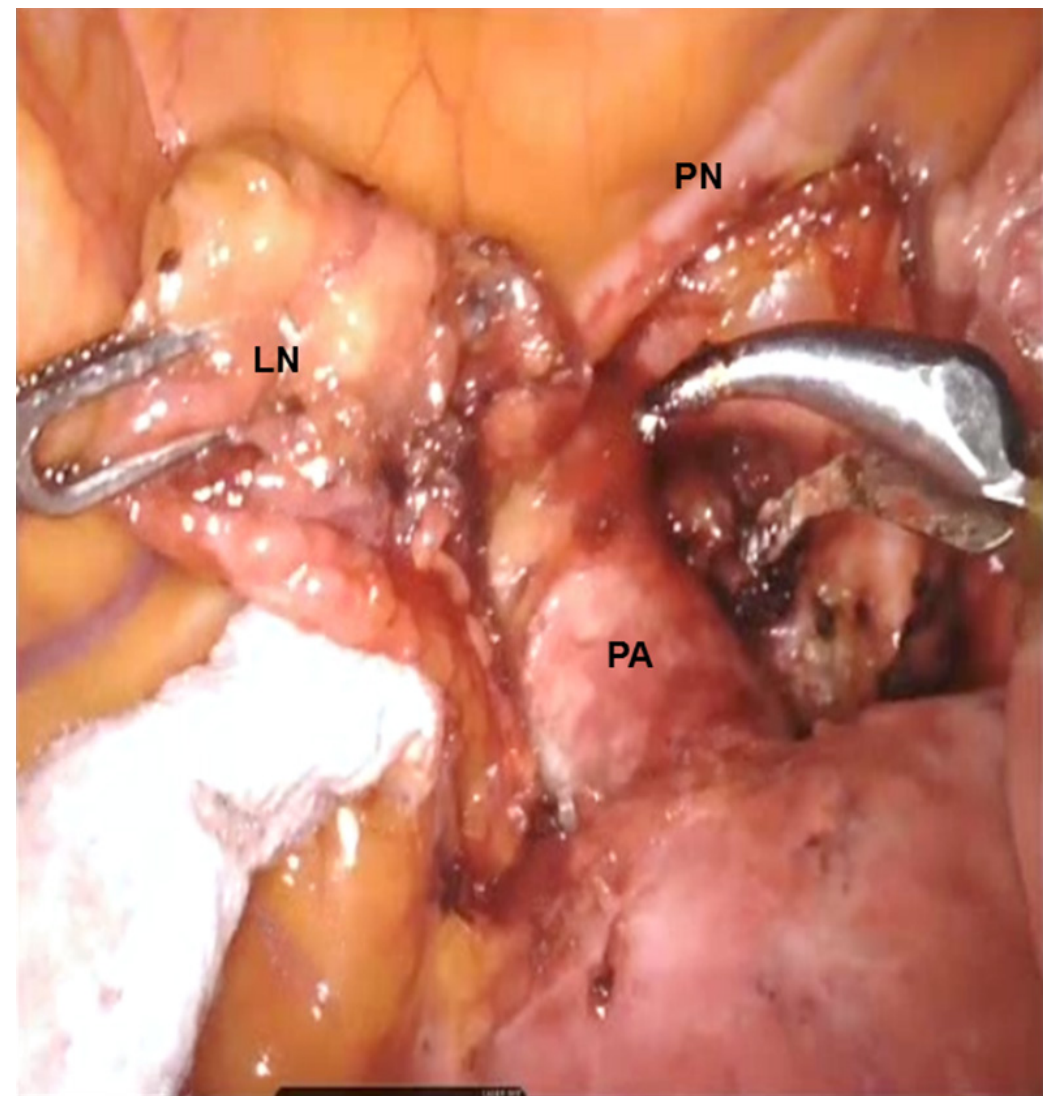

Figure 12. LS3 Segmentectomy: the nodes in station \#5 (LN) are removed and the proximal left PA is exposed just posterior to the left PN. PA: pulmonary artery; PN: phrenic nerve; LN: lymph node

\section{Left upper lobe anterior anatomic segmentectomy (S3)}

Following the dissection of mediastinal nodes, the lung is retracted posteriorly and the anterior hilum is approached. The nodes in station $\# 5$ are removed and the proximal left pulmonary artery is exposed just posterior to the left phrenic nerve [Figure 12]. The nodes between the superior pulmonary vein and the pulmonary artery are dissected and removed. The superior pulmonary vein is separated from the underlying pulmonary artery [Figure 13]. Figure 14 shows the anatomic relationship among the vein, artery, and bronchus in segment $\mathrm{S}_{3}(\mathrm{~V} 3, \mathrm{~A} 3$ and $\mathrm{B} 3)$.

$\mathrm{V} 3$ is encircled, elevated with a vessel loop, and divided with a stapler with a white cartridge. Care is taken to preserve the $\mathrm{V}_{1}$ branch to the $\mathrm{S}_{1}$ segment of the upper lobe. The B3 bronchus is encircled, elevated off the pulmonary artery, and divided with a stapler using a purple cartridge. Division of the B3 facilitates division of the A3 PA branch(es). The A3 PA branch is encircled with a vessel loop and divided with a stapling device. The A3 PA branches can be divided before dividing B3; however, this usually requires suture ligation and division of the A3. Next the intersegmental fissures between $\mathrm{S}_{1}+\mathrm{S} 2$ and $\mathrm{S} 3$ and between $\mathrm{S} 4+\mathrm{S} 5$ and S3 are delineated either using indocyanine green if using the Xi robot or inflation technique and divided using a stapler carrying a green cartridge [Figure 15].

\section{Left upper lobe apical and posterior anatomic segmentectomy (S1 + S2)}

The approach to these left sided segments is similar. Although individual posterior (S2) segmentectomy is possible, instead of an individual apical segmentectomy, many times an apicoposterior $\left(\mathrm{S}_{1}+\mathrm{S}_{2}\right)$ segmentectomy is performed on the left side. 


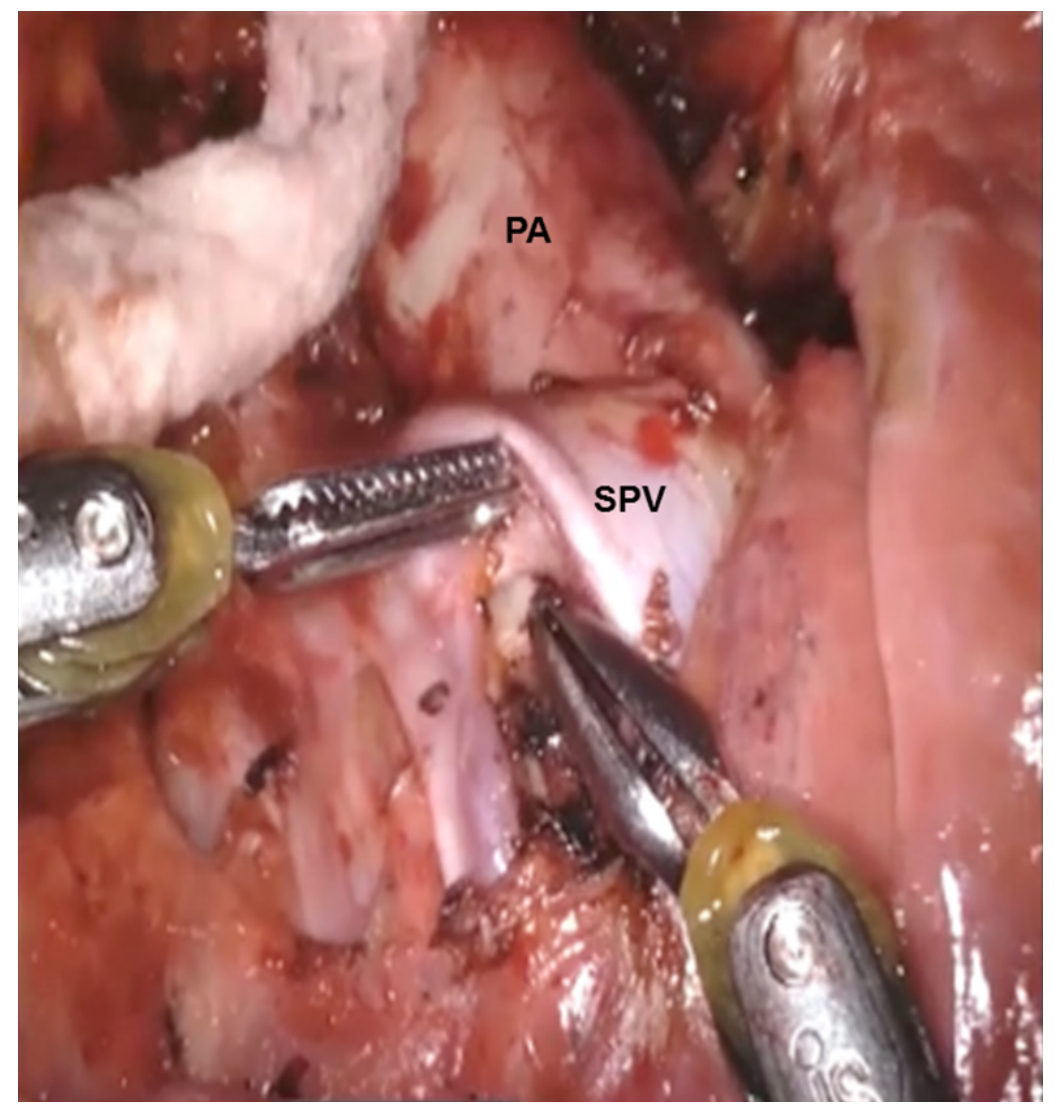

Figure 13. LS3 Segmentectomy: the nodes between the SPV and the PA are dissected and removed. PA: pulmonary artery; SPV: superior pulmonary vein

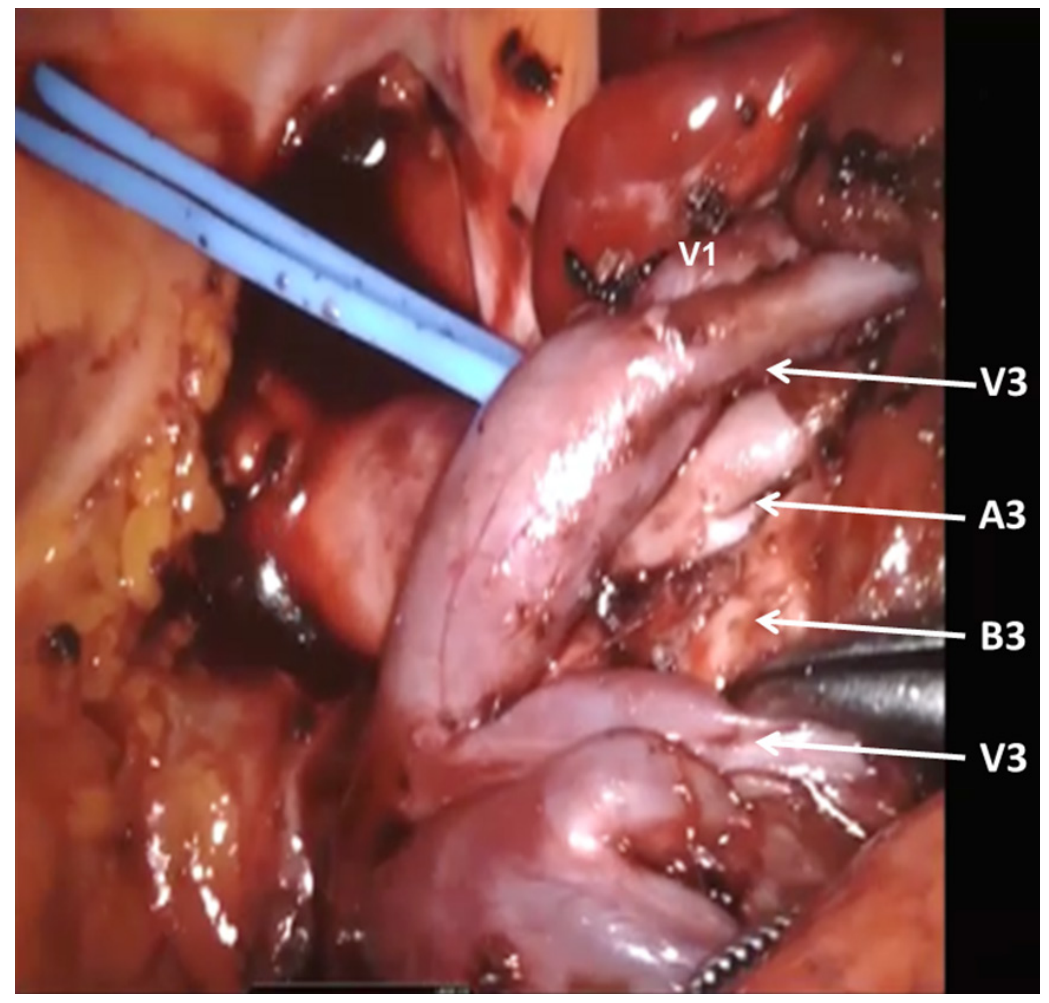

Figure 14. LS3 Segmentectomy: the anatomic relationship between S3 segmental veins (V3), S3 segmental artery (A3), and S3 segmental bronchus (B3) 


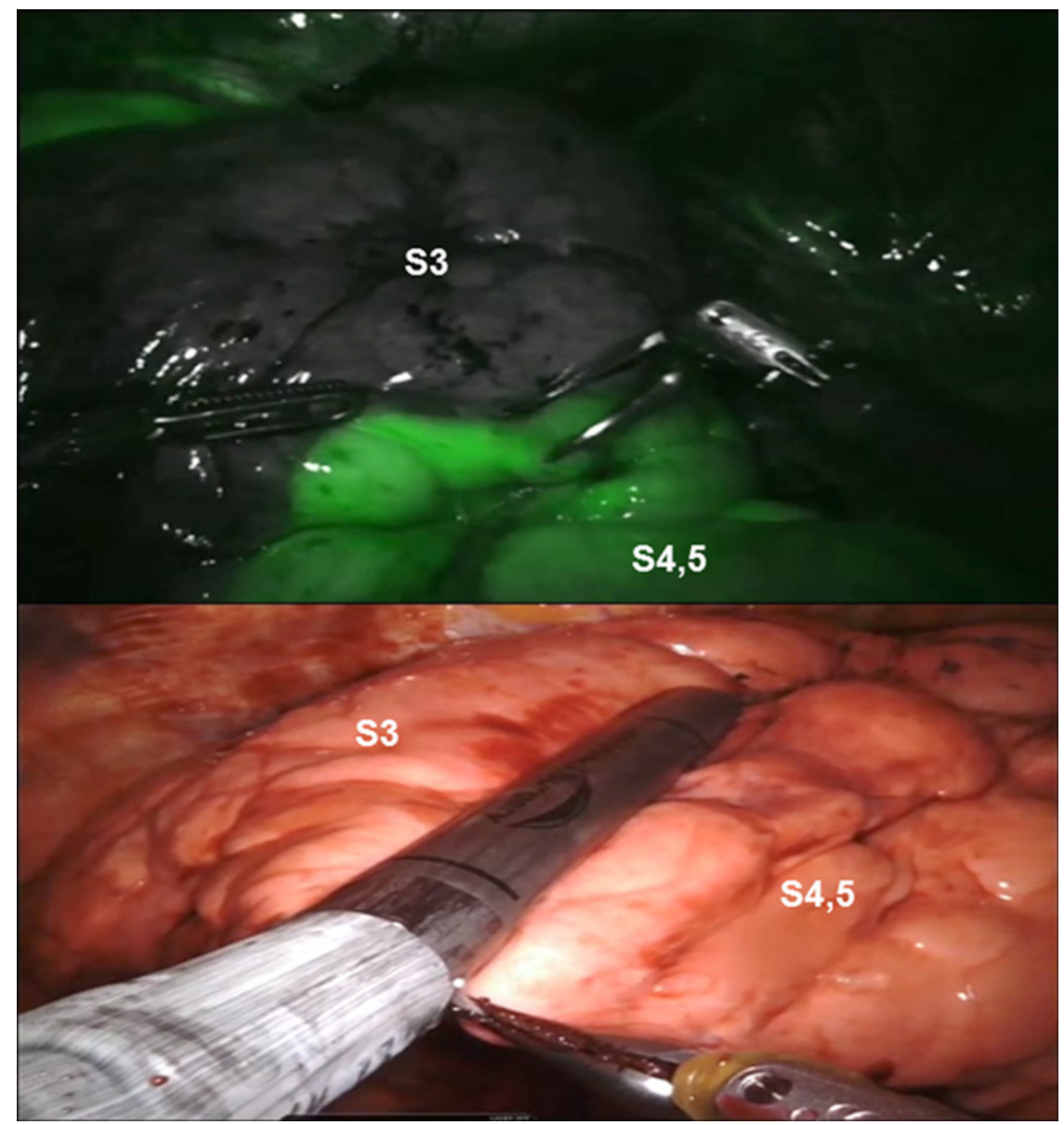

Figure 15. LS3 Segmentectomy: the intersegmental fissure between S3, and S4, 5 is delineated either by using Indocyanine Green if using the Xi robot or by inflation if using the Si robot

As with all segmentectomies, the procedure begins with mediastinal nodal dissection as has been described previously.

For a posterior $\mathrm{S}_{2}$ or apicoposterior $\mathrm{S}_{1}+\mathrm{S}_{2}$ segmentectomy, the pulmonary artery branches to the respective segments as identified in Figure 16. The branches are encircled, elevated with a vessel loop, and divided with a vascular stapler carrying a white load. Following the division of the pulmonary artery branches, the bronchus is approached from the back. The segmental bronchus is isolated, the N1 nodes are excised, and the bronchus is encircled and divided with a stapler with a purple or blue cartridge [Figure 17]. For these segments, the segmental veins are usually taken with division of the fissure. The intersegmental fissure is identified as has been outlined previously and divided in a stepwise progressive manner using a stapling device with a green cartridge [Figure 18].

\section{Left upper lobe lingulectomy and anatomic segmentectomy (S4 + S5)}

Lingulectomy can be performed with either a vein first or artery first technique. The advantage of the artery first technique is that the fissure is approached first, station \#11 nodes are removed first, and if they are positive, a left upper lobectomy is performed.

After a complete mediastinal nodal dissection as with the other left sided segmentectomies, the oblique fissure is opened and the subadventitial plane above the descending pulmonary artery is entered [Figure 19]. The "V" shaped space between the lower lobe pulmonary artery and the Lingular artery is dissected and all $\mathrm{N} 1$ nodes are removed. 


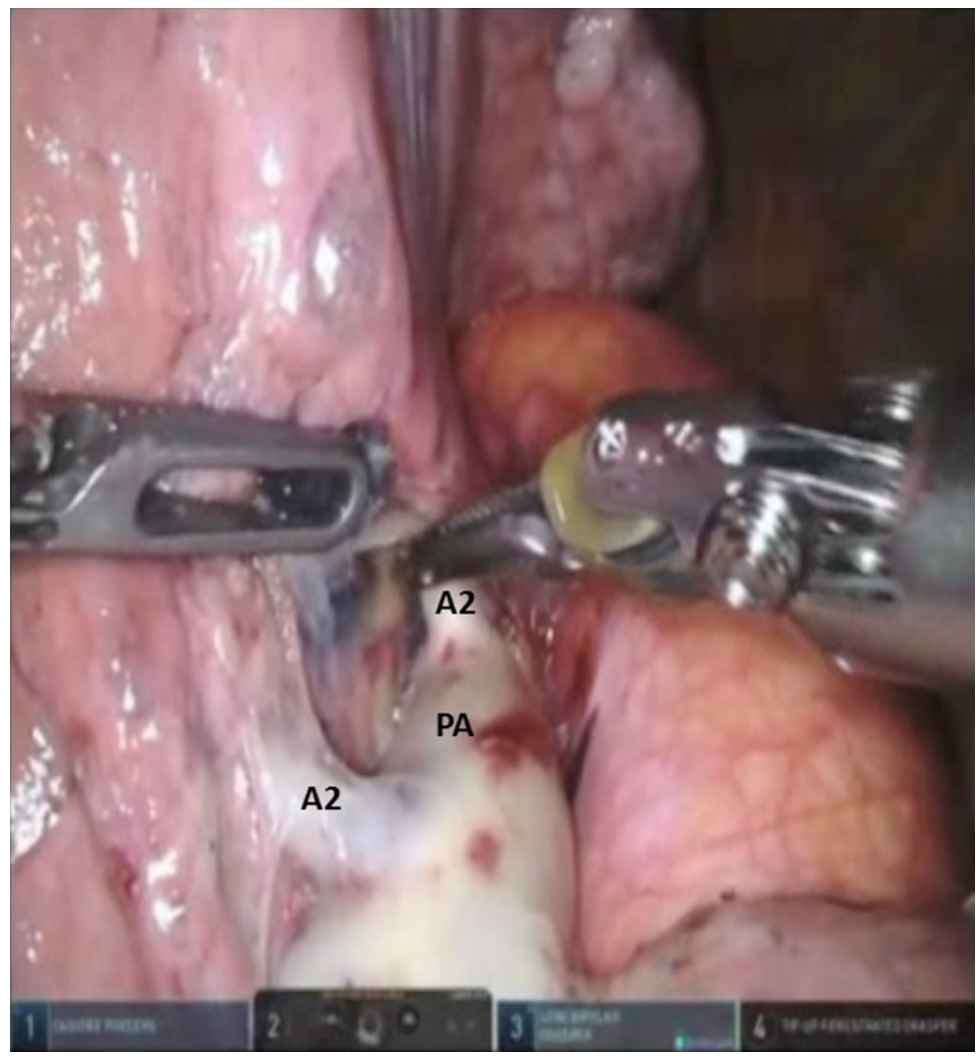

Figure 16. LS1, S2 Segmentectomy: the pulmonary artery branch to the posterior segment (A2) are identified. PA: pulmonary artery

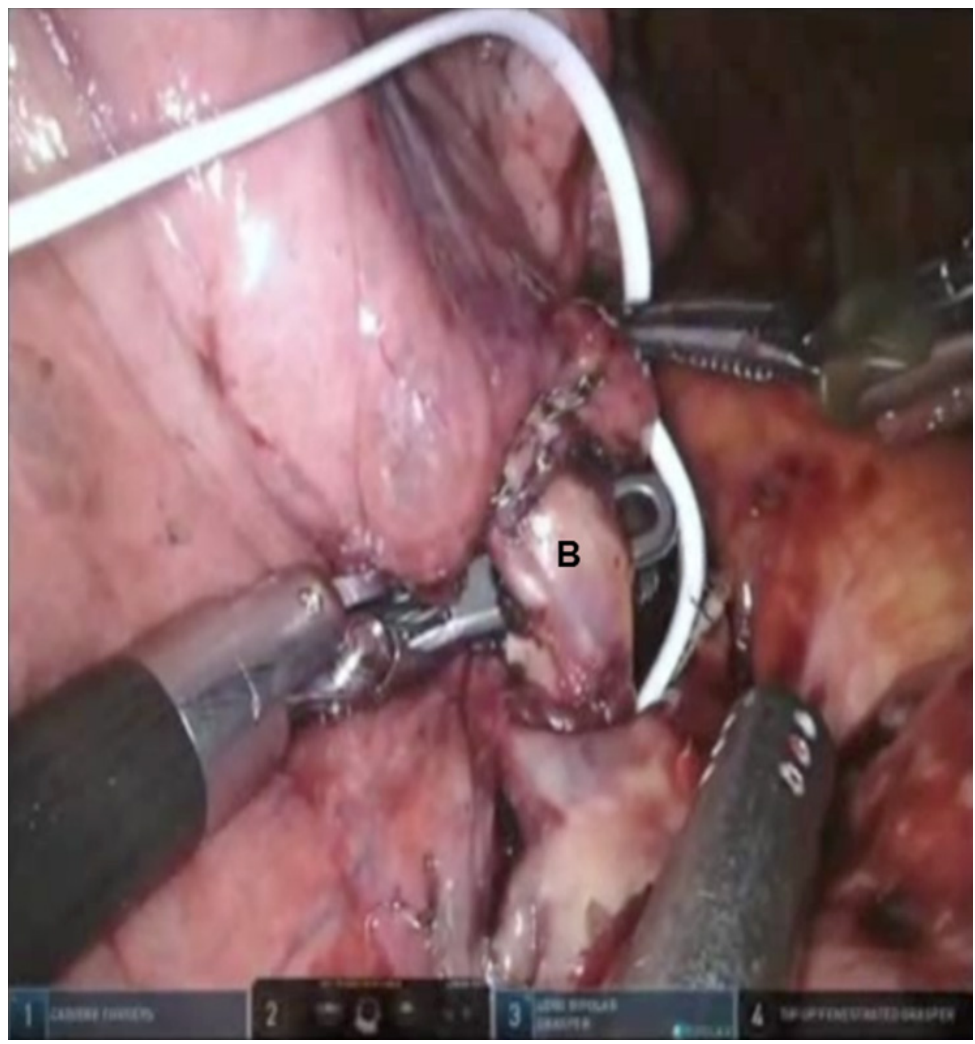

Figure 17. LS1, S2 Segmentectomy: the segmental bronchus (B) is isolated and divided 


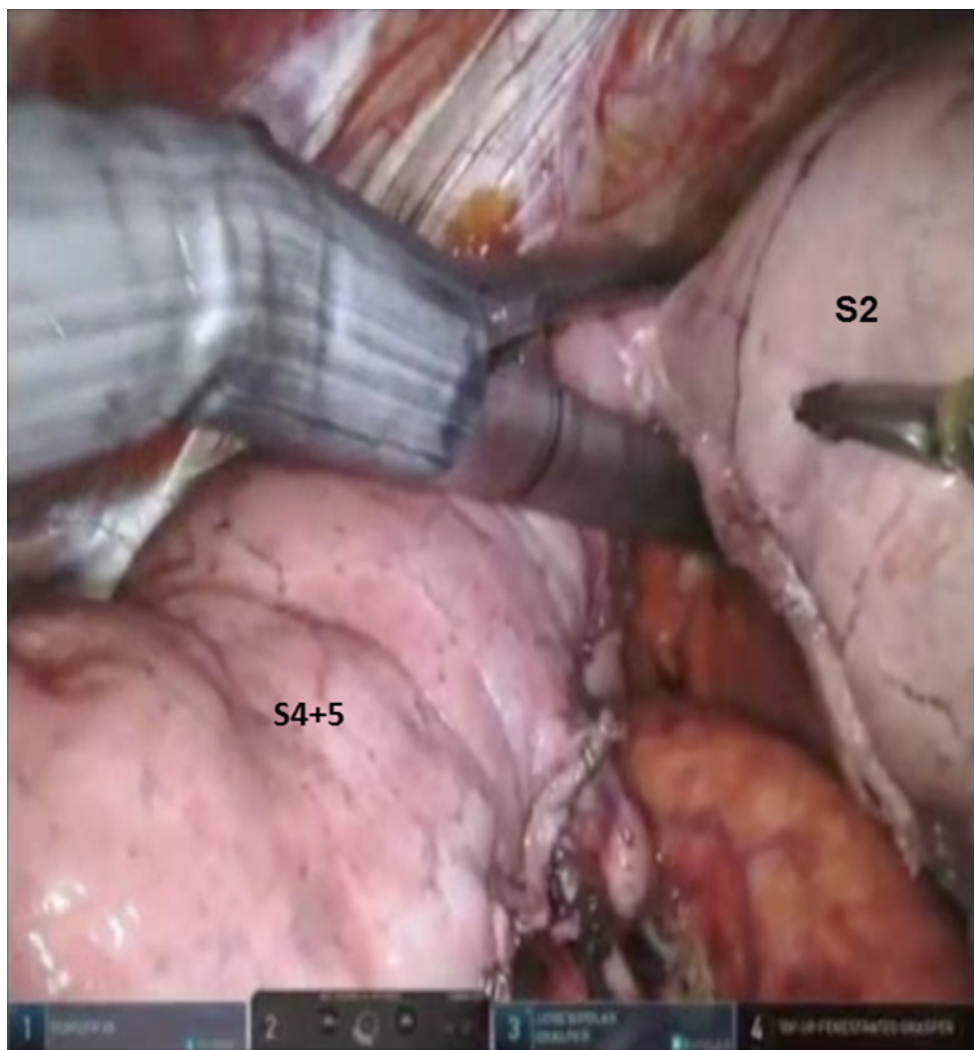

Figure 18. LS1, S2 Segmentectomy: the intersegmental fissure between S1 + S2 and S4 + S5 segments is divided in a stepwise progressive manner using a stapling device with a Green load

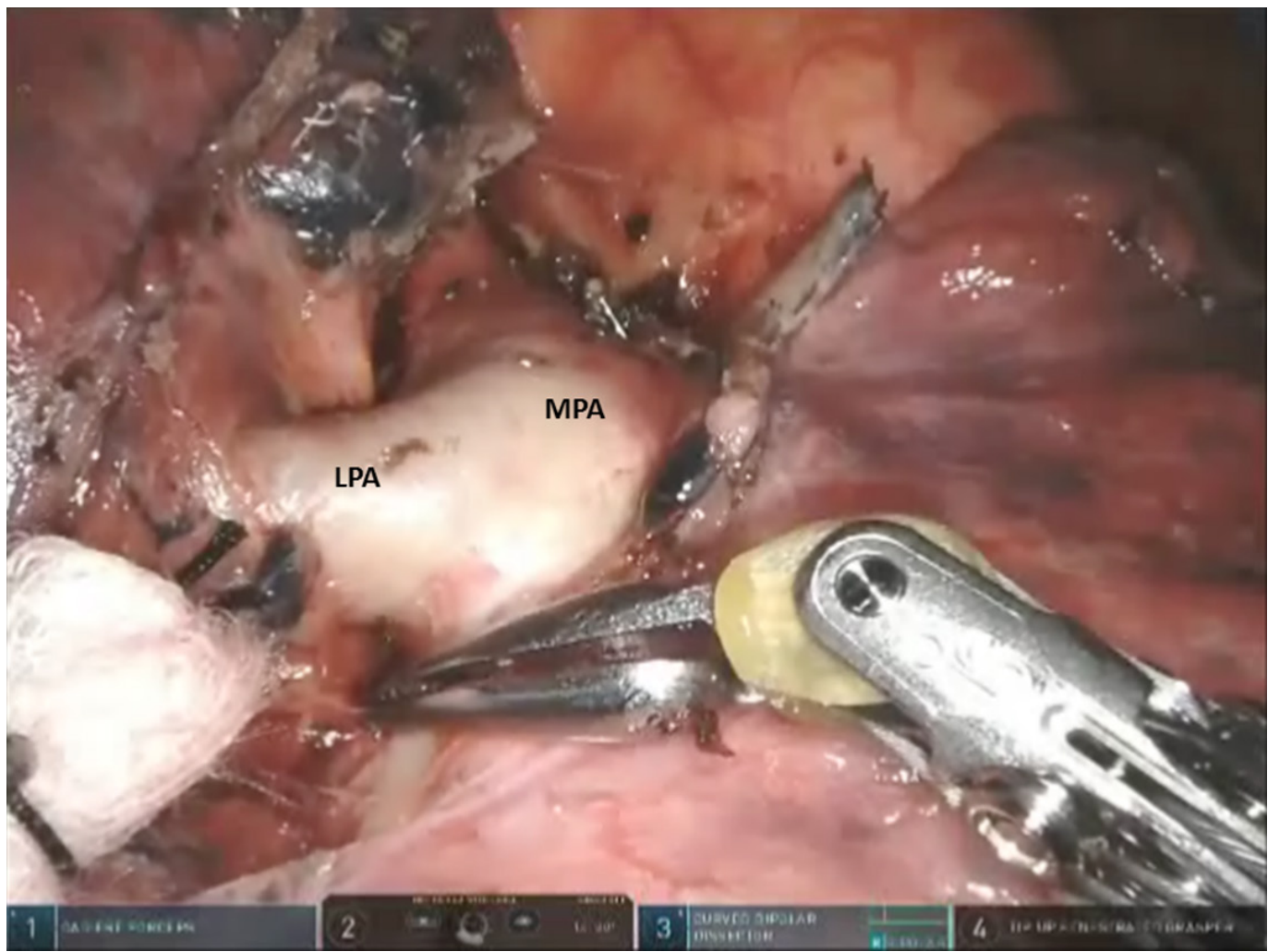

Figure 19. Lingulectomy (LS4, LS5): the oblique fissure is opened and the subadventitial plane above the descending pulmonary artery is entered. MPA: main pulmonary artery; LPA: lingular pulmonary artery 


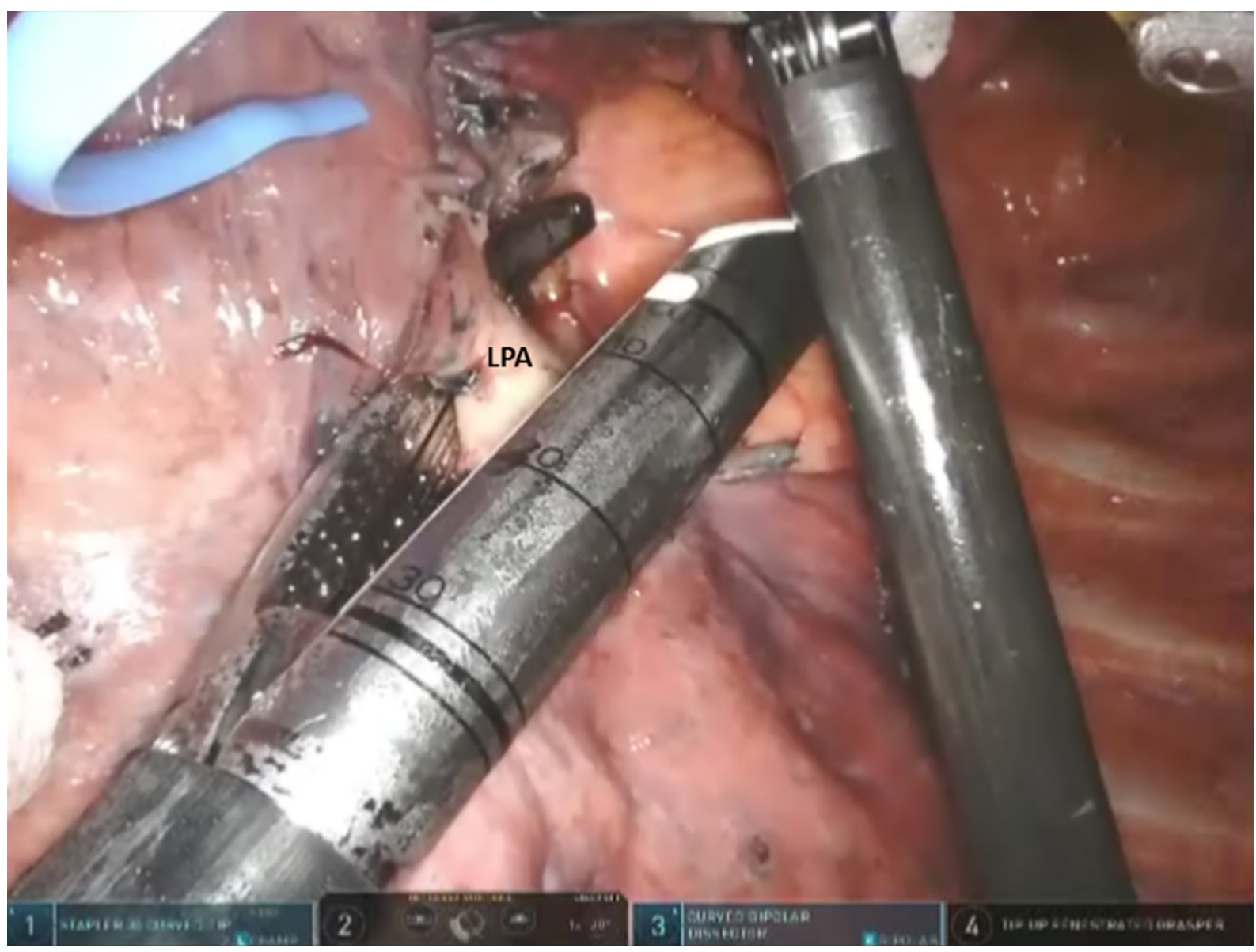

Figure 20. Lingulectomy (LS4, LS5): LPA is encircled and elevated with a vessel loop and divided with a stapler carrying a white cartridge. LPA: lingular pulmonary artery

Next, the lung is retracted posteriorly, and the anterior hilum is approached. The space between the superior and inferior pulmonary veins is developed and the nodes are removed. The superior pulmonary vein is dissected away from the underlying pulmonary artery, encircled with a vessel loop, and elevated. After the entire superior pulmonary vein is dissected, the Lingular vein(s) are identified, encircled, elevated with a vessel loop, and divided with a vascular stapler. Then, the anterior aspect of the oblique fissure is divided by passing a stapler with a blue cartridge from an anterior to posterior direction, heading toward the space between the Lingular artery and the inferior pulmonary artery. This enables easy access to the Lingular pulmonary artery which is encircled, elevated with a vessel loop, and divided with a stapler carrying a white cartridge [Figure 20]. Division of the fissure also enables access to the Lingular bronchus. The Lingular bronchus is encircled and elevated with a vessel loop; the anesthesiologist removes any indwelling suction catheters and the bronchus is divided with a stapler using a green cartridge [Figure 21]. Finally, using the techniques which have been outlined earlier, the intersegmental fissure between $\mathrm{S}_{1}+\mathrm{S}_{2}$, S3, and the Lingula are identified [Figure 22]. The lung parenchyma is then divided with multiple firings of a stapling device with a blue or green cartridge.

\section{Robotic left lower lobe anatomic superior segmentectomy (S6)}

Port placement and instruments are similar to the left upper lobe segmentectomy procedures.

Following the complete mediastinal dissection which has been outlined previously, the pulmonary artery is identified in the oblique fissure. The subadventitial plane overlying the pulmonary artery is entered, and 


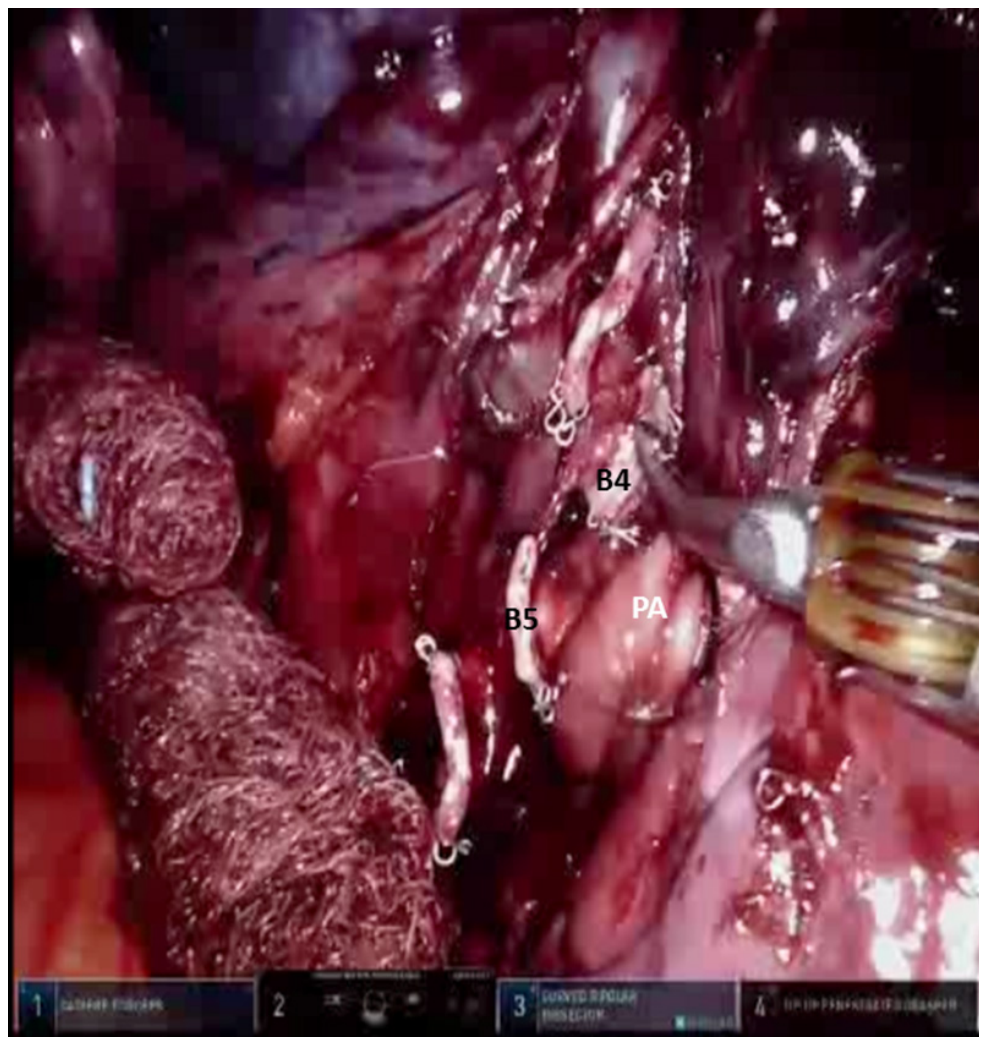

Figure 21. Lingulectomy (LS4, LS5): the stump of the Lingular bronchus is seen (B4, B5). PA: main pulmonary artery

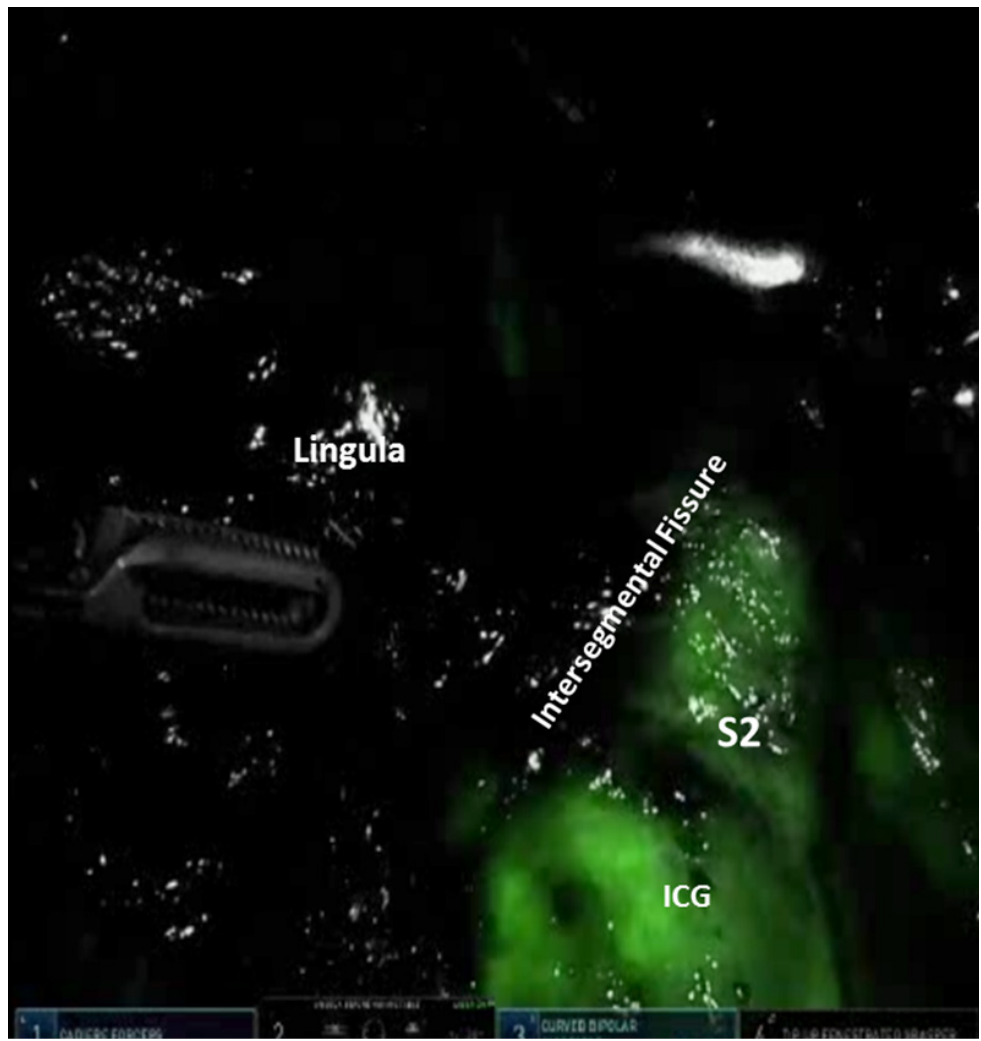

Figure 22. Lingulectomy (LS4, LS5): the intersegmental fissure between S2 and the Lingula is identified using ICG dye. ICG: indocyanine green 


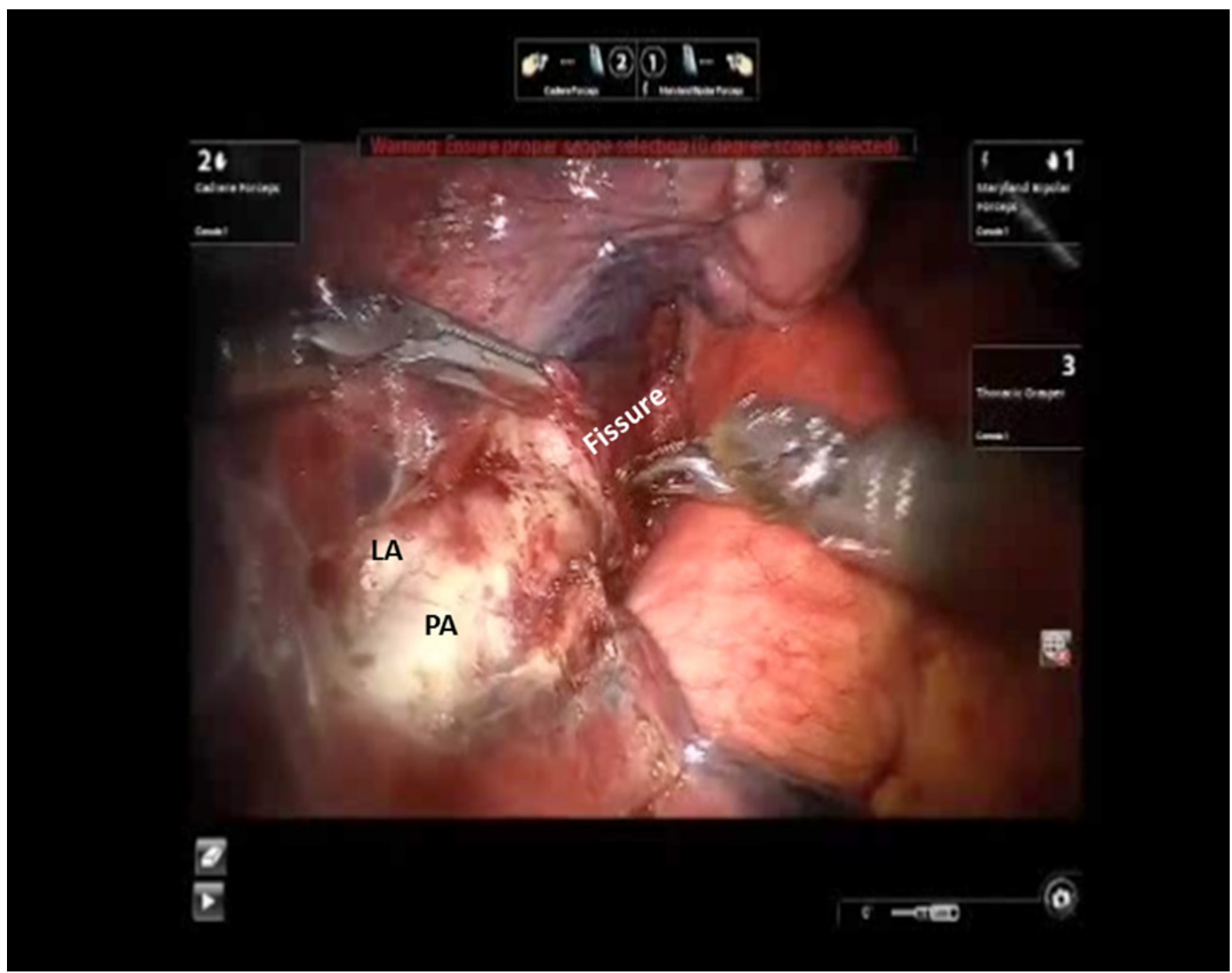

Figure 23. LS6 Segmentectomy: the subadventitial plane is entered, and dissection is carried posteriorly under the pulmonary parenchyma in the posterior aspect of the fissure toward the main pulmonary artery. LA: lingular artery; PA: descending main pulmonary artery

dissection is carried posteriorly under the pulmonary parenchyma in the posterior aspect of the fissure toward the main pulmonary artery [Figure 23]. A pair of Cadiere forceps is used to pass a vessel loop under the pulmonary parenchyma in the posterior aspect of the fissure. A stapler with a blue cartridge is then used to divide the tissue in the posterior aspect of the fissure. The subadventitial plane is then developed anteriorly in order to identify the descending branch of the pulmonary artery. The anterior aspect of the oblique fissure is divided. The superior segmental pulmonary artery is identified. The Cadiere forceps is passed under the superior segmental pulmonary artery, a vessel loop is passed underneath and used to encircle and elevate the vessel, and the vessel is divided with a stapler with a white vascular cartridge introduced from a medial to lateral direction [Figure 24].

The lung is elevated and retracted medially. The Cadiere forceps is passed from a medial to lateral direction under the inferior pulmonary vein and a vessel loop is used to encircle and elevate the vein [Figure 25]. The superior segmental vein is identified, encircled, and divided using a stapler with a white vascular cartridge introduced from inferior to superior direction [Figure 26]. The nodes overlying the left lower lobe bronchus are swept toward the specimen. The B6 bronchus is identified, encircled, and divided [Figure 27]. The intersegmental fissure between the S6 and the basal segments of the lower lobe is identified as has been outline previously and divided using a stapling device. 


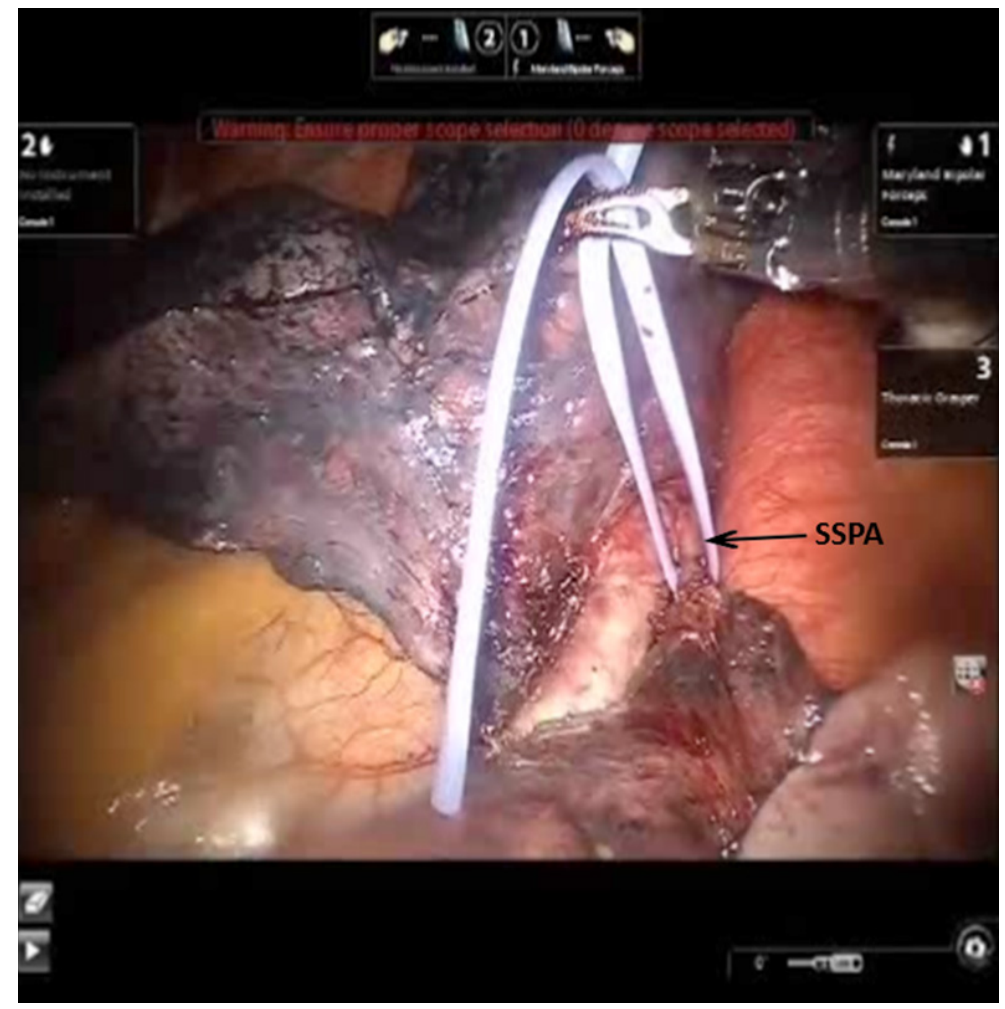

Figure 24. LS6 Segmentectomy: a vessel loop is passed underneath SSPA and used to encircle and elevate the vessel. SSPA: superior segmental pulmonary artery

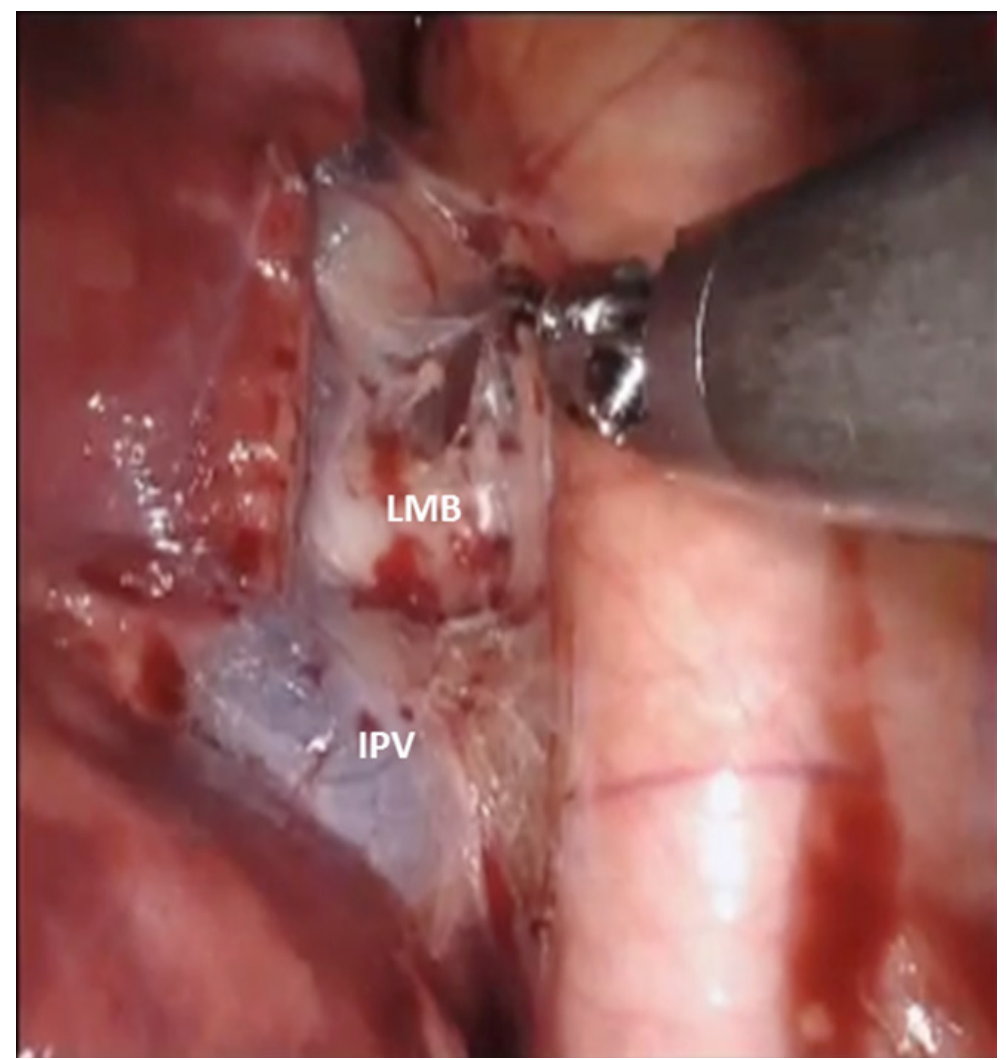

Figure 25. LS7-LS10 Segmentectomy: the IPV is isolated. LMB: left mainstem bronchus; IPV: inferior pulmonary vein 


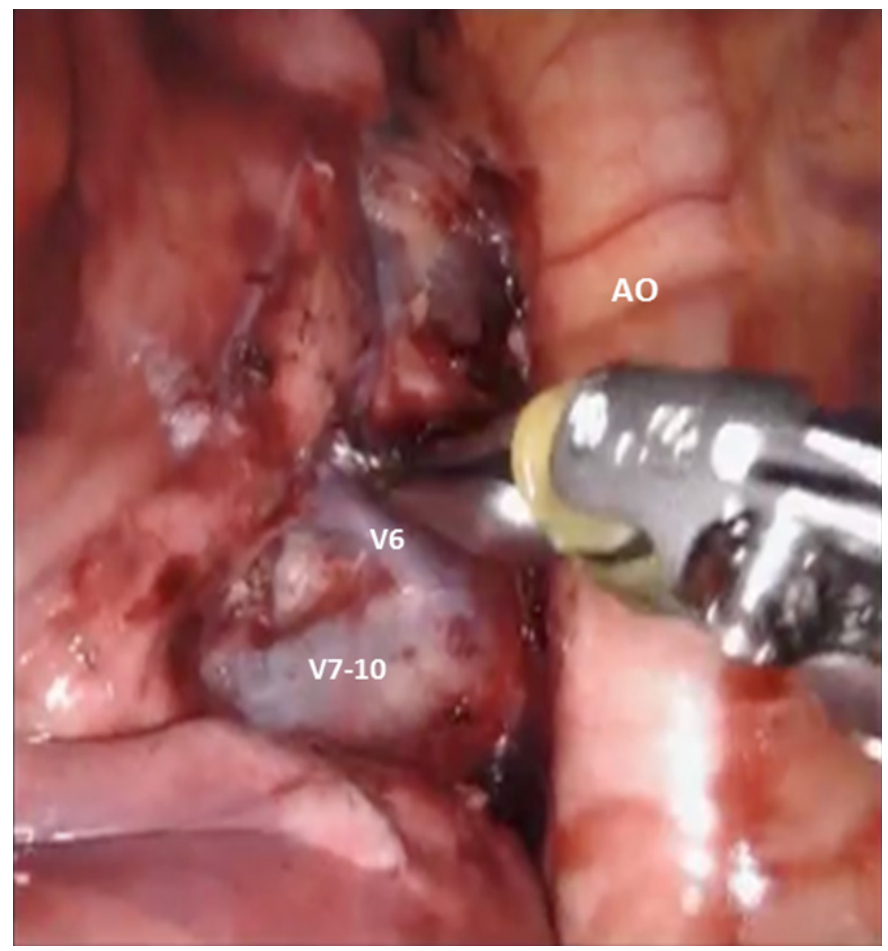

Figure 26. LS7-LS10 Segmentectomy: isolation of the left lower lobe basal vein (V7-10) from superior segmental vein (V6). AO: aorta

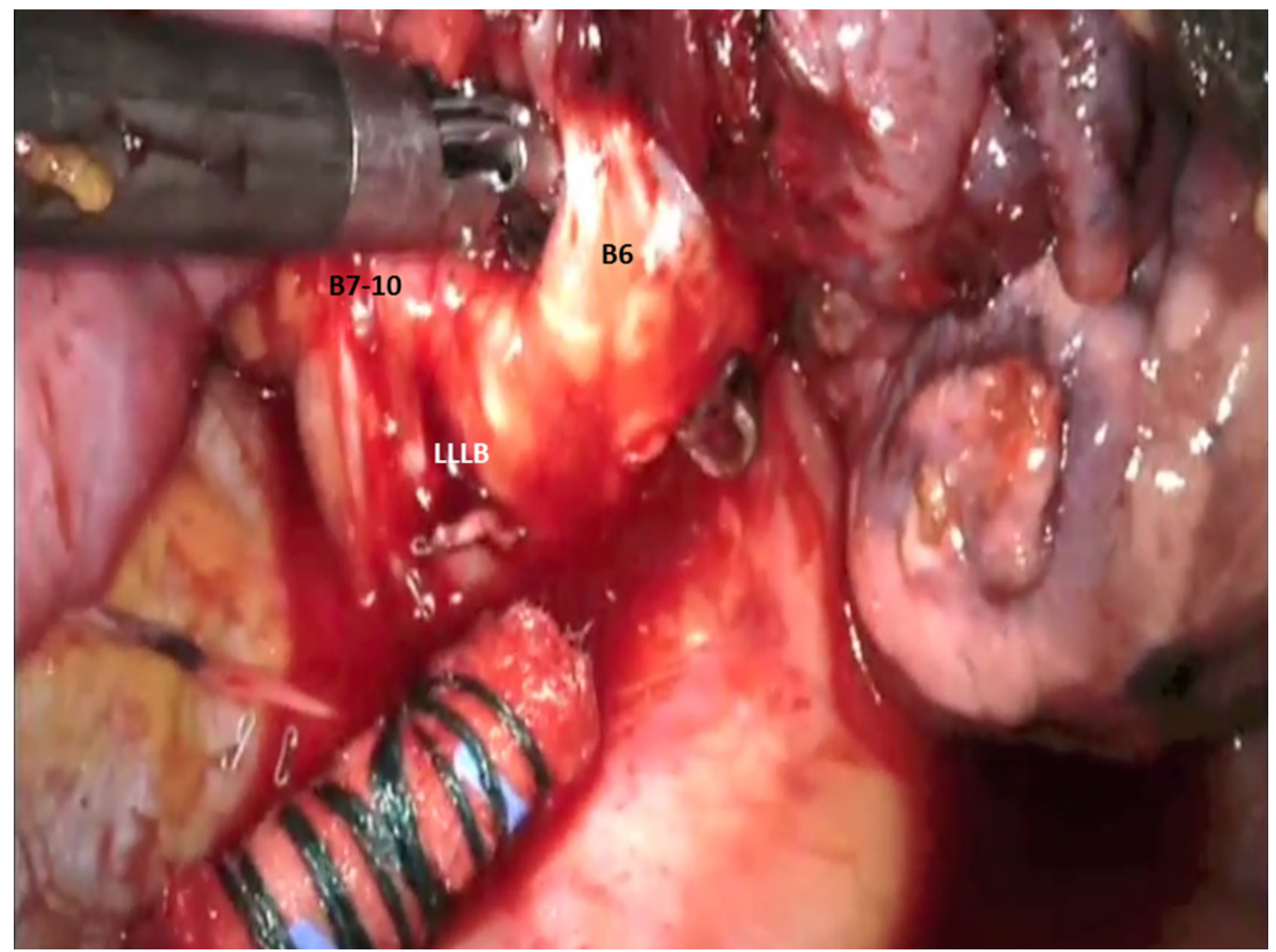

Figure 27. LS6 Segmentectomy: B6 bronchus is identified, encircled, and divided. LLLB: left lower lobe bronchus; B7-10: bronchus to basal segment of left lower lobe 


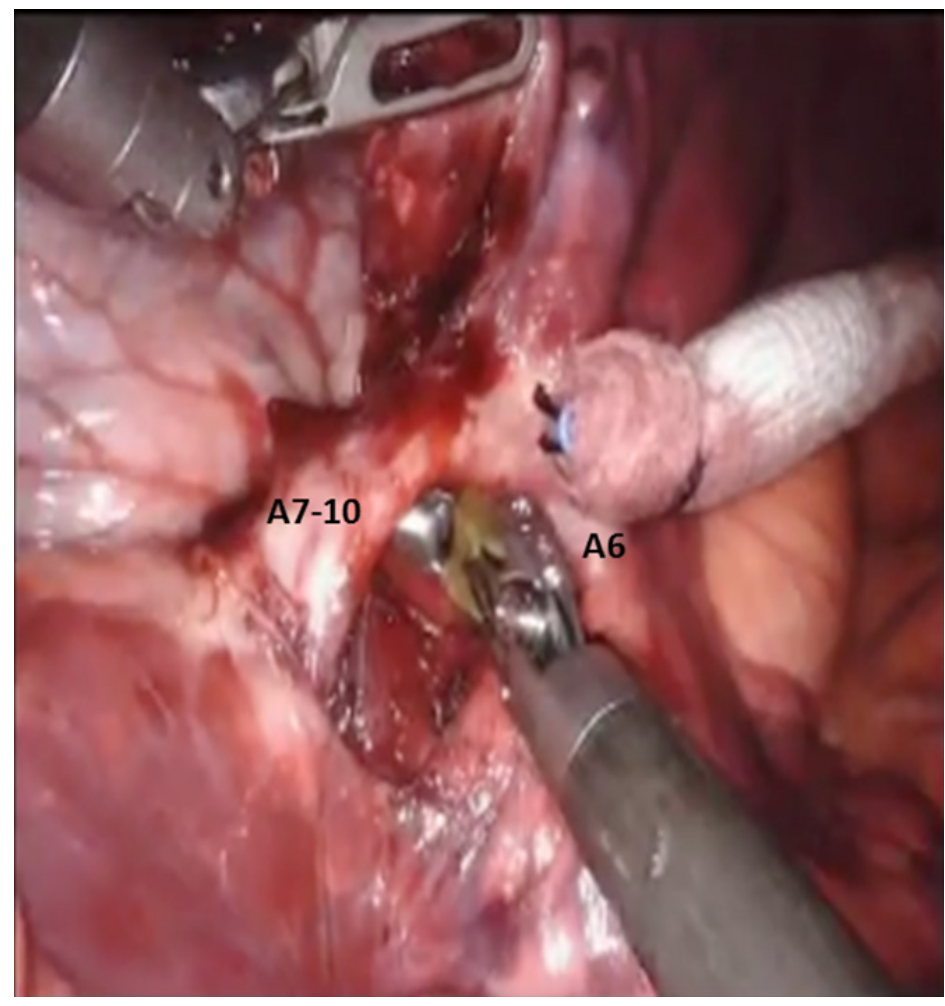

Figure 28. LS7-LS10 Segmentectomy: the basal branch of the left pulmonary (A7-10) is encircled and divided. A6: pulmonary to superior segment of left lower lobe (S6)

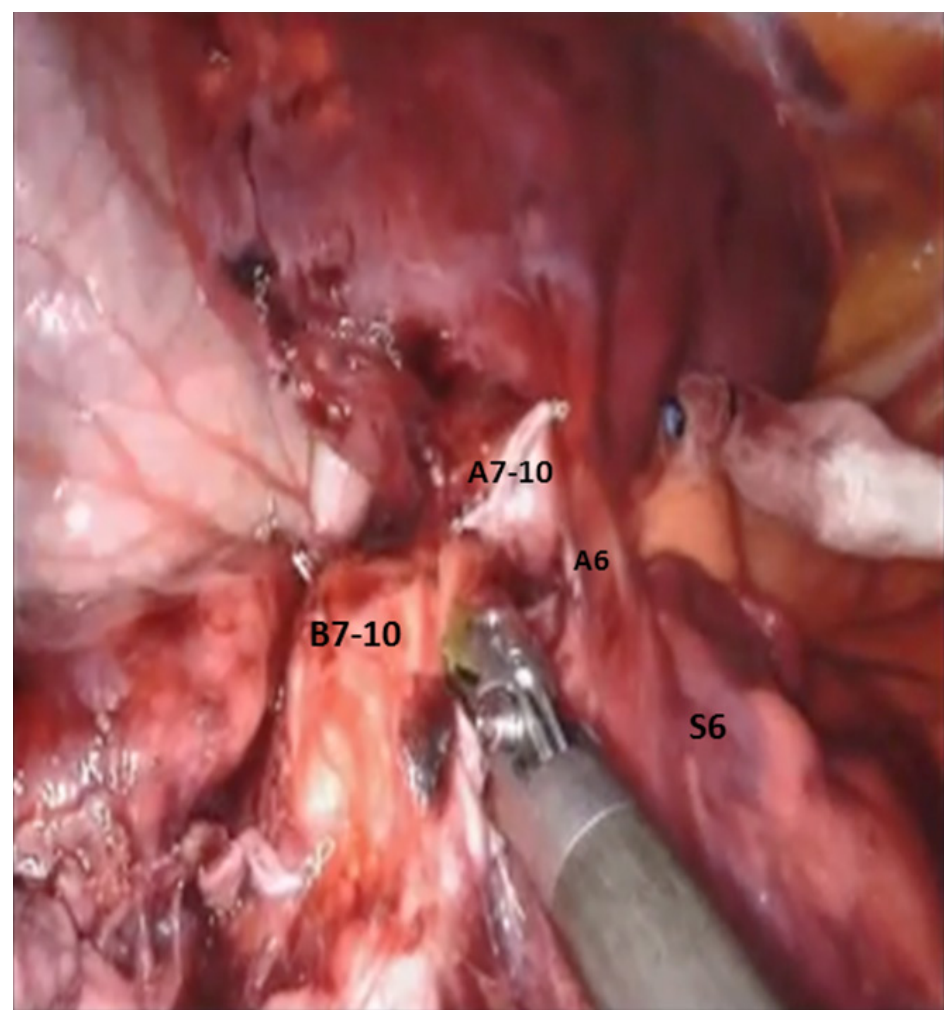

Figure 29. LS7-LS10 Segmentectomy: the bronchus to the basal segment (B7-10) is encircled and divided. A7-10: stump pf the divided pulmonary artery branch to basal segment; A6: pulmonary artery branch to superior segment of left lower lobe (S6); S6: superior segment of left lower lobe 


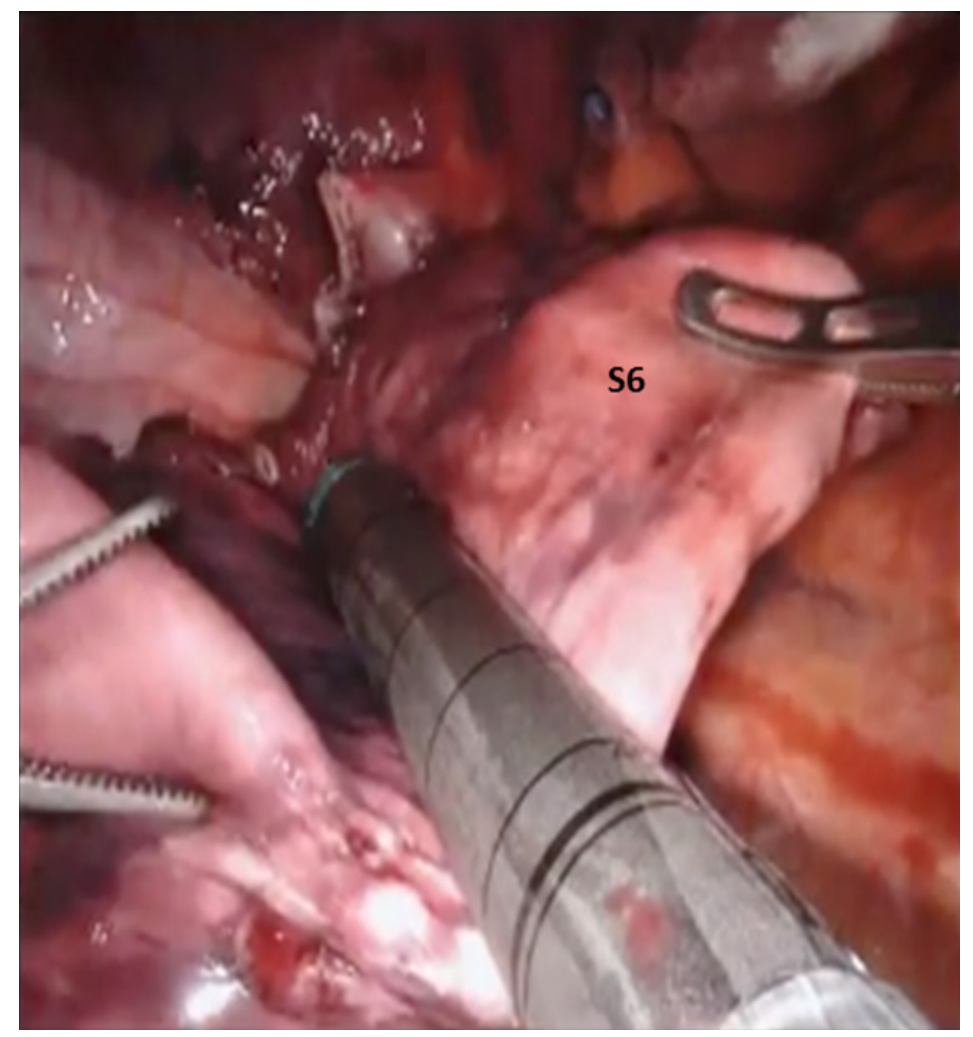

Figure 30. LS7-LS10 Segmentectomy: the intersegmental fissure is identified and divided using a stapler with a green cartridge

\section{Robotic left lower lobe anatomic basal segmentectomy (S7-S10)}

The approach to this segmentectomy is similar to superior segmentectomy (S6). Following the complete mediastinal nodal dissection, the inferior pulmonary vein is encircled with a vessel loop and elevated. Then the superior segmental vein is identified, thereby allowing for identification of the basal branch of the inferior pulmonary vein. The basal vein $(\mathrm{V} 7-10)$ is then divided with a stapling device with a white cartridge. Next, the pulmonary artery is isolated in the fissure as has been described previously. The left lower lobe pulmonary artery is identified [Figure 28]. The basal branch of the left pulmonary artery is encircled and elevated with a vessel loop and divided with a vascular stapler. Following the division of the A7-10, the bronchus to the basal segment (B7-10) is encircled and divided with a stapler carrying a blue cartridge [Figure 29]. Finally the intersegmental fissure is identified and divided using a stapler with a green cartridge [Figure 30].

\section{CONCLUSION}

Anatomic pulmonary segmentectomy in patients with early stage lung cancer is an oncologically efficacious procedure. The surgical robot allows for precise dissection of the segmental bronchopulmonary structures while minimizing trauma to surrounding tissues, and it allows for thorough and complete dissection of the mediastinal nodes. Robotic segmentectomy should be considered when planning a lung sparing operation in patients with small tumors, in elderly patients or patients with borderline lung function.

\section{DECLARATIONS}

\section{Authors' contributions}

Contributed equally to the performance of the surgeries, collection of data and writing the manuscript: Gharagozloo F, Meyer M 


\section{Availability of data and materials}

Not applicable.

\section{Financial support and sponsorship}

None.

\section{Conflicts of interest}

Both authors declared that there are no conflicts of interest.

\section{Ethical approval and consent to participate}

Not applicable.

\section{Consent for publication}

Not applicable.

\section{Copyright}

(c) The Author(s) 2020.

\section{REFERENCES}

1. Jackson CM. Morris's human anatomy. 8th ed. Philadelphia: P. Blakiston's Sons \& Co.; 1925.

2. Churchill ED, Belsey R. Segmental pneumonectomy in bronchiectasis. Ann Surg 1939;109:481-99.

3. Brock RC. The nomenclature of broncho-pulmonary anatomy: an international nomenclature accepted by the thoracic society. Thorax 1950;5:222-8.

4. Walcott-Sapp S, Sukumar M. The history of pulmonary lobectomy: two phases of innovation. CTS net, 2016. Available from: https:// www.ctsnet.org/article/history-pulmonary-lobectomy-two-phases-innovation. [Last accessed on 3 Aug 2020]

5. Sienel W, Dango S, Kirschbaum A, Cucuruz B, Stremmel WHC, et al. Sublobar resections in stage IA non-small cell lung cancer: segmentectomies result in significantly better cancer-related survival than wedge resection. Eur J Cardiothorac Surg 2008;33:728-34.

6. Miller DL, Rowland CM, Deschamps C, Allen MS, Trastek VF, et al. Surgical treatment of non-small cell lung cancer 1 cm or less in diameter. Ann Thorac Surg 2002;73:1545-50.

7. Yang CF, D'Amico TA. Thoracoscopic segmentectomy for lung cancer. Ann Thorac Surg 2012;94:668-81.

8. Shapiro M, Weiser TS, Wisnivesky JP, Chin C, Arustamyan M, et al. Thoracoscopic segmentectomy compares favorably with thoracoscopic lobectomy for patients with small stage I lung cancer. J Thorac Cardiovasc Surg 2009;137:1388-93.

9. D'Amico TA. Thoracoscopic segmentectomy: technical considerations and outcomes. Ann Thorac Surg 2008;85:S716-8.

10. Leshnower BG, Miller DL, Fernandez FG, Pickens A, Force SD. Video-assisted thoracoscopic surgery segmentectomy: a safe and effective procedure. Ann Thorac Surg 2010;89:1571-6.

11. Nomori H. Anatomical segmentectomy for clinical stage IA non-small cell lung cancer. Nihon Geka Gakkai Zasshi 2011;112:264-6. (in Japanese)

12. Dai C, Shen J, Ren Y, Zhong S, Zheng H, et al. Choice of surgical procedure for patients with non-small-cell lung cancer $\leq 1 \mathrm{~cm}$ or $>1$ to $2 \mathrm{~cm}$ among lobectomy, segmentectomy, and wedge resection: a population-based study. J Clin Oncol 2016;34:3175-82.

13. Narsule CK, Ebright MI, Fernando HC. Sublobar versus lobar resection: current status. Cancer J 2011;17:23-7.

14. Hayasaka K, Shiono S, Suzuki K, Endoh M. Long-term results of sublobar resection for lung cancer;a 10-year follow-up study. Kyobu Geka 2019;72:23-9. (in Japanese)

15. Nguyen D, Gharagozloo F, Tempesta B, Meyer M, Gruessner A. Long-term results of robotic anatomical segmentectomy for early-stage non-small-cell lung cancer. Eur J Cardiothorac Surg 2019;55:427-33.

16. Migliore M. Robotic assisted lung resection needs further evidence. J Thorac Dis 2016;8:E1274-8. 